\title{
Search for lepton flavour violation in ep collisions at HERA
}

\section{Journal Article}

\section{Author(s):}

H1 Collaboration; Aktas, A.; Berger, Niklaus; Del Degan, Marc A.; Grab, Christoph; Leibenguth, Guillaume; Sauter, Michel; Schöning, André; Weber, Ronald; Zimmermann, Tobias; et al.

\section{Publication date:}

2007-12

\section{Permanent link:}

https://doi.org/10.3929/ethz-b-000004929

\section{Rights / license:}

Creative Commons Attribution 4.0 International

\section{Originally published in:}

The European Physical Journal C 52(4), https://doi.org/10.1140/epjc/s10052-007-0440-2 


\section{Search for lepton flavour violation in ep collisions at HERA}

\section{The H1 Collaboration}

A. Aktas ${ }^{11}$, C. Alexa ${ }^{11,49}$, V. Andreev ${ }^{25}$, T. Anthonis ${ }^{4,5}$, B. Antunovic ${ }^{26}$, S. Aplin ${ }^{11}$, A. Asmone ${ }^{33}$, A. Astvatsatourov ${ }^{4,5}$, A. Babaev ${ }^{24, \dagger}$, S. Backovic ${ }^{30}$, A. Baghdasaryan ${ }^{38}$, P. Baranov ${ }^{25}$, E. Barrelet ${ }^{29}$, W. Bartel ${ }^{11}$, S. Baudrand ${ }^{27}$, M. Beckingham ${ }^{11}$, K. Begzsuren ${ }^{35}$, O. Behnke ${ }^{14}$, O. Behrendt ${ }^{8}$, A. Belousov ${ }^{25}$, N. Berger ${ }^{40}$, J.C. Bizot ${ }^{27}$, M.-O. Boenig 8 , V. Boudry ${ }^{28}$, I. Bozovic-Jelisavcic ${ }^{2}$, J. Bracinik ${ }^{26}$, G. Brandt ${ }^{14}$, M. Brinkmann ${ }^{11}$, V. Brisson ${ }^{27}$, D. Bruncko ${ }^{16}$, F.W. Büsser ${ }^{12}$, A. Bunyatyann ${ }^{13,38}$, G. Buschhorn ${ }^{26}$, L. Bystritskaya ${ }^{24}$, A.J. Campbell ${ }^{11}$, K.B. Cantun Avila ${ }^{22}$, F. Cassol-Brunner ${ }^{21}$, K. Cerny ${ }^{32}$, V. Cerny ${ }^{16,47}$, V. Chekelian ${ }^{26}$, A. Cholewa ${ }^{11}$, J.G. Contreras ${ }^{22}$, J.A. Coughlan ${ }^{6}$, G. Cozzika ${ }^{10}$, J. Cvach ${ }^{31}$, J.B. Dainton ${ }^{18}$, K. Daum ${ }^{37,43}$, Y. de Boer ${ }^{24}$, B. Delcourt ${ }^{27}$, M. Del Degan ${ }^{40}$, A. De Roeck ${ }^{11,45}$, E.A. De Wolf 4,5 , C. Diaconu ${ }^{21, a}$, V. Dodonov ${ }^{13}$, A. Dubak ${ }^{30,46}$, G. Eckerlin ${ }^{11}$, V. Efremenko ${ }^{24}$, S. Egli ${ }^{36}$, R. Eichler ${ }^{36}$, F. Eisele ${ }^{14}$, A. Eliseev ${ }^{25}$, E. Elsen ${ }^{11}$, S. Essenov ${ }^{24}$, A. Falkewicz ${ }^{7}$, P.J.W. Faulkner ${ }^{3}$, L. Favart ${ }^{4,5}$, A. Fedotov ${ }^{24}$, R. Felst ${ }^{11}$, J. Feltesse ${ }^{10,48}$, J. Ferencei ${ }^{16}$, L. Finke ${ }^{11}$, M. Fleischer ${ }^{11}$, A. Fomenko ${ }^{25}$, G. Franke ${ }^{11}$, T. Frisson ${ }^{28}$, E. Gabathuler ${ }^{18}$, E. Garutti ${ }^{11}$, J. Gayler ${ }^{11}$, S. Ghazaryan ${ }^{38}$, S. Ginzburgskaya ${ }^{24}$, A. Glazov ${ }^{11}$, I. Glushkov ${ }^{39}$, L. Goerlich ${ }^{7}$, M. Goettlich ${ }^{11}$, N. Gogitidze ${ }^{25}$, S. Gorbounov ${ }^{39}$, M. Gouzevitch ${ }^{28}$, C. Grab ${ }^{40}$, T. Greenshaw ${ }^{18}$, M. Gregori ${ }^{19}$, B.R. Grell ${ }^{11}$, G. Grindhammer ${ }^{26}$, S. Habib ${ }^{12, b}$, D. Haidt ${ }^{11}$, M. Hansson ${ }^{20}$, G. Heinzelmann ${ }^{12}$, C. Helebrant ${ }^{11}$, R.C.W. Henderson ${ }^{17}$, H. Henschel ${ }^{39}$, G. Herrera ${ }^{23}$, M. Hildebrandt ${ }^{36}$, K.H. Hiller ${ }^{39}$, D. Hoffmann ${ }^{21}$, R. Horisberger ${ }^{36}$, A. Hovhannisyan ${ }^{38}$, T. Hreus ${ }^{4,5,44}$, S. Hussain ${ }^{19}$, M. Jacquet ${ }^{27}$, M.E. Janssen ${ }^{11}$, X. Janssen ${ }^{4,5}$, V. Jemanov ${ }^{12}$, L. Jönsson ${ }^{20}$, D.P. Johnson ${ }^{4,5}$, A.W. Jung ${ }^{15}$, H. Jung ${ }^{11}$, M. Kapichine ${ }^{9}$, J. Katzy ${ }^{11}$, I.R. Kenyon ${ }^{3}$, C. Kiesling ${ }^{26}$, M. Klein ${ }^{39}$, C. Kleinwort ${ }^{11}$, T. Klimkovich ${ }^{11}$, T. Kluge ${ }^{11}$, G. Knies ${ }^{11}$, A. Knutsson ${ }^{20}$, V. Korbel ${ }^{11}$, P. Kostka ${ }^{39}$, M. Kraemer ${ }^{11}$, K. Krastev ${ }^{11}$, J. Kretzschmar ${ }^{39}$, A. Kropivnitskaya ${ }^{24}$, K. Krüger ${ }^{15}$, M.P.J. Landon ${ }^{19}$, W. Lange ${ }^{39}$, G. Laštovička-Medin ${ }^{30}$, P. Laycock ${ }^{18}$,

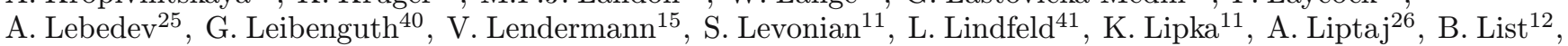
J. List ${ }^{11}$, N. Loktionova ${ }^{25}$, R. Lopez-Fernandez ${ }^{23}$, V. Lubimov ${ }^{24}$, A.-I. Lucaci-Timoce ${ }^{11}$, H. Lueders ${ }^{12}$, L. Lytkin ${ }^{13}$, A. Makankine ${ }^{9}$, E. Malinovski ${ }^{25}$, P. Marage ${ }^{4,5}$, L. Marti ${ }^{11}$, M. Martisikova ${ }^{11}$, H.-U. Martyn ${ }^{1}$, S.J. Maxfield ${ }^{18}$, A. Mehta ${ }^{18}$, K. Meier ${ }^{15}$, A.B. Meyer ${ }^{11}$, H. Meyer ${ }^{11}$, H. Meyer ${ }^{37}$, J. Meyer ${ }^{11}$, V. Michels ${ }^{11}$, S. Mikocki ${ }^{7}$, I. Milcewicz-Mika ${ }^{7}$, D. Mladenov ${ }^{34}$, A. Mohamed ${ }^{18}$, F. Moreau ${ }^{28}$, A. Morozov ${ }^{9}$, J.V. Morris ${ }^{6}$, M.U. Mozer ${ }^{14}$, K. Müller ${ }^{41}$, P. Murín ${ }^{16,44}$, K. Nankov ${ }^{34}$, B. Naroska ${ }^{12}$, T. Naumann ${ }^{39}$, P.R. Newman ${ }^{3}$, C. Niebuhr ${ }^{11}$,

A. Nikiforov ${ }^{26}$, G. Nowak $^{7}$, K. Nowak ${ }^{41}$, M. Nozicka ${ }^{39}$, R. Oganezov ${ }^{38}$, B. Olivier ${ }^{26}$, J.E. Olsson ${ }^{11}$, S. Osman ${ }^{20}$,

D. Ozerov ${ }^{24}$, V. Palichik ${ }^{9}$, I. Panagoulias ${ }^{11,42, c}$, M. Pandurovic ${ }^{2}$, T. Papadopoulou ${ }^{11,42, c}$, C. Pascaud ${ }^{27}$,

G.D. Patel ${ }^{18}$, H. Peng ${ }^{11}$, E. Perez ${ }^{10}$, D. Perez-Astudillo ${ }^{22}$, A. Perieanu ${ }^{11}$, A. Petrukhinn ${ }^{24}$, I. Picuric ${ }^{30}$, S. Piec $^{39}$, D. Pitzl ${ }^{11}$, R. Plačakyté ${ }^{11}$, B. Povh ${ }^{13}$, T. Preda ${ }^{11,49}$, P. Prideaux ${ }^{18}$, A.J. Rahmat ${ }^{18}$, N. Raicevic ${ }^{30}$, T. Ravdandorj ${ }^{35}$, P. Reimer ${ }^{31}$, A. Rimmer ${ }^{18}$, C. Risler ${ }^{11}$, E. Rizvi ${ }^{19}$, P. Robmann ${ }^{41}$, B. Roland ${ }^{4,5}$, R. Roosen ${ }^{4,5}$, A. Rostovtsev ${ }^{24}$, Z. Rurikova ${ }^{11}$, S. Rusakov ${ }^{25}$, F. Salvaire ${ }^{11}$, D.P.C. Sankey ${ }^{6}$, M. Sauter ${ }^{40}$, E. Sauvan ${ }^{21}$, S. Schmidt ${ }^{11}$, S. Schmitt ${ }^{11}$, C. Schmitz ${ }^{41}$, L. Schoeffel ${ }^{10}$, A. Schöning ${ }^{40}$, H.-C. Schultz-Coulon ${ }^{15}$, F. Sefkow ${ }^{11}$, R.N. Shaw-West ${ }^{3}$, I. Sheviakov ${ }^{25}$, L.N. Shtarkov ${ }^{25}$, T. Sloan ${ }^{17}$, I. Smiljanic ${ }^{2}$, P. Smirnov ${ }^{25}$, Y. Soloviev ${ }^{25}$, D. South ${ }^{8}$, V. Spaskov ${ }^{9}$, A. Specka ${ }^{28}$, M. Steder ${ }^{11}$, B. Stella ${ }^{33}$, J. Stiewe ${ }^{15}$, U. Straumann ${ }^{41}$, D. Sunar ${ }^{4,5}$, T. Sykora ${ }^{4,5}$, V. Tchoulakov ${ }^{9}$, G. Thompson ${ }^{19}$, P.D. Thompson ${ }^{3}$, T. Toll ${ }^{11}$, F. Tomasz ${ }^{16}$, D. Traynor ${ }^{19}$, T.N. Trinh ${ }^{21}$, P. Truöl ${ }^{41}$, I. Tsakov ${ }^{34}$, B. Tseepeldorj ${ }^{35}$, G. Tsipolitis ${ }^{11,42}$, I. Tsurin ${ }^{39}$, J. Turnau ${ }^{7}$, E. Tzamariudaki ${ }^{26}$, K. Urban ${ }^{15}$, A. Usik ${ }^{25}$, D. Utkin ${ }^{24}$, A. Valkárová ${ }^{32}$, C. Vallée $e^{21}$, P. Van Mechelen ${ }^{4,5}$, A. Vargas Trevino ${ }^{11}$, Y. Vazdik ${ }^{25}$, S. Vinokurova ${ }^{11}$, V. Volchinski ${ }^{38}$, K. Wacker ${ }^{8}$, G. Weber ${ }^{12}$, R. Weber ${ }^{40}$, D. Wegener ${ }^{8}$, C. Werner ${ }^{14}$, M. Wessels ${ }^{11}$, C. Wissing ${ }^{11}$, R. Wolf ${ }^{14}$, E. Wünsch $^{11}$, S. Xella ${ }^{41}$, W. Yan ${ }^{11}$, V. Yeganov ${ }^{38}$, J. Žáček ${ }^{32}$, J. Zálešák ${ }^{31}$, Z. Zhang ${ }^{27}$, A. Zhelezov ${ }^{24}$, A. Zhokin²4, Y.C. Zhu ${ }^{11}$, J. Zimmermann ${ }^{26}$, T. Zimmermann ${ }^{40}$, H. Zohrabyan ${ }^{38}$, F. Zomer ${ }^{27}$

${ }^{1}$ I. Physikalisches Institut der RWTH, Aachen, Germany ${ }^{\mathrm{d}}$

${ }^{2}$ Vinca Institute of Nuclear Sciences, Belgrade, Serbia

${ }^{3}$ School of Physics and Astronomy, University of Birmingham, Birmingham, UK ${ }^{\mathrm{e}}$

${ }^{4}$ Inter-University Institute for High Energies ULB-VUB, Brussels, Belgium

${ }^{5}$ Universiteit Antwerpen, Antwerpen, Belgium ${ }^{\mathrm{f}}$

${ }_{7}^{6}$ Rutherford Appleton Laboratory, Chilton, Didcot, UK ${ }^{\mathrm{e}}$

${ }^{7}$ Institute for Nuclear Physics, Cracow, Poland ${ }^{\mathrm{g}}$

${ }^{8}$ Institut für Physik, Universität Dortmund, Dortmund, Germany ${ }^{\mathrm{d}}$

9 Joint Institute for Nuclear Research, Dubna, Russia

${ }^{10}$ CEA, DSM/DAPNIA, CE-Saclay, Gif-sur-Yvette, France 
11 DESY, Hamburg, Germany

${ }^{12}$ Institut für Experimentalphysik, Universität Hamburg, Hamburg, Germany ${ }^{\mathrm{d}}$

13 Max-Planck-Institut für Kernphysik, Heidelberg, Germany

14 Physikalisches Institut, Universität Heidelberg, Heidelberg, Germany ${ }^{\mathrm{d}}$

${ }^{15}$ Kirchhoff-Institut für Physik, Universität Heidelberg, Heidelberg, Germany ${ }^{\mathrm{d}}$

16 Institute of Experimental Physics, Slovak Academy of Sciences, Košice, Slovak Republic ${ }^{\mathrm{h}}$

17 Department of Physics, University of Lancaster, Lancaster, UK ${ }^{\mathrm{e}}$

18 Department of Physics, University of Liverpool, Liverpool, UK ${ }^{\mathrm{e}}$

19 Queen Mary and Westfield College, London, UK ${ }^{\mathrm{e}}$

20 Physics Department, University of Lund, Lund, Sweden ${ }^{\mathrm{i}}$

${ }^{21}$ CPPM, CNRS/IN2P3 - Univ. Mediterranee, Marseille, France

22 Departamento de Fisica Aplicada, CINVESTAV, Mérida, Yucatán, México ${ }^{\mathrm{j}}$

23 Departamento de Fisica, CINVESTAV, México ${ }^{\mathrm{k}}$

${ }^{24}$ Institute for Theoretical and Experimental Physics, Moscow, Russia ${ }^{\mathrm{k}}$

25 Lebedev Physical Institute, Moscow, Russia ${ }^{1}$

26 Max-Planck-Institut für Physik, München, Germany

27 LAL, Université de Paris-Sud 11, IN2P3-CNRS, Orsay, France

28 LLR, Ecole Polytechnique, IN2P3-CNRS, Palaiseau, France

29 LPNHE, Universités Paris VI and VII, IN2P3-CNRS, Paris, France

${ }^{30}$ Faculty of Science, University of Montenegro, Podgorica, Montenegro ${ }^{\mathrm{i}}$

${ }^{31}$ Institute of Physics, Academy of Sciences of the Czech Republic, Praha, Czech Republic ${ }^{\mathrm{m}}$

32 Faculty of Mathematics and Physics, Charles University, Praha, Czech Republic ${ }^{\mathrm{m}}$

33 Dipartimento di Fisica Università di Roma Tre and INFN Roma 3, Roma, Italy

34 Institute for Nuclear Research and Nuclear Energy, Sofia, Bulgaria ${ }^{i}$

35 Institute of Physics and Technology of the Mongolian Academy of Sciences, Ulaanbaatar, Mongolia

36 Paul Scherrer Institut, Villigen, Switzerland

37 Fachbereich C, Universität Wuppertal, Wuppertal, Germany

38 Yerevan Physics Institute, Yerevan, Armenia

39 DESY, Zeuthen, Germany

40 Institut für Teilchenphysik, ETH, Zürich, Switzerland ${ }^{\mathrm{n}}$

41 Physik-Institut der Universität Zürich, Zürich, Switzerland ${ }^{\mathrm{n}}$

42 Also at Physics Department, National Technical University, Zografou Campus, 15773 Athens, Greece

43 Also at Rechenzentrum, Universität Wuppertal, Wuppertal, Germany

44 Also at University of P.J. Šafárik, Košice, Slovak Republic

45 Also at CERN, Geneva, Switzerland

46 Also at Max-Planck-Institut für Physik, München, Germany

47 Also at Comenius University, Bratislava, Slovak Republic

48 Also at DESY and University Hamburg, Helmholtz Humboldt Research Award, Germany

49 Also at National Institute for Physics and Nuclear Engineering, Magurele, Bucharest, Romania

$\dagger$ deceased

Received: 3 March 2007 / Revised version: 26 September 2007 /

Published online: 24 October 2007 - (C) Springer-Verlag / Società Italiana di Fisica 2007

Abstract. A search for the lepton flavour violating processes $e p \rightarrow \mu X$ and $e p \rightarrow \tau X$ is performed with the H1 experiment at HERA. Final states with a muon or tau and a hadronic jet are searched for in a data sample corresponding to an integrated luminosity of $66.5 \mathrm{pb}^{-1}$ for $e^{+} p$ collisions and $13.7 \mathrm{pb}^{-1}$ for $e^{-} p$ collisions at a centre-of-mass energy of $319 \mathrm{GeV}$. No evidence for lepton flavour violation is found. Limits are derived on the mass and the couplings of leptoquarks inducing lepton flavour violation in an extension of the Buchmüller-Rückl-Wyler effective model. Leptoquarks produced in ep collisions with a coupling strength of $\lambda=0.3$ and decaying with the same coupling strength to a muon-quark pair or a tau-quark pair are excluded at $95 \%$ confidence level up to masses of $459 \mathrm{GeV}$ and $379 \mathrm{GeV}$, respectively.

\footnotetext{
a e-mail: diaconu@cppm.in2p3.fr

b Supported by a scholarship of the World Laboratory Björn Wiik Research Project

c This project is co-funded by the European Social Fund (75\%) and National Resources (25\%) - (EPEAEK II) PYTHAGORAS II
}

\footnotetext{
d Supported by the Bundesministerium für Bildung und Forschung, FRG, under contract numbers 05 H1 1GUA/1, 05 H1 1PAA/1, 05 H1 1PAB/9, 05 H1 1PEA/6, 05 H1 1VHA/7 and $05 \mathrm{H} 11 \mathrm{VHB} / 5$

e Supported by the UK Particle Physics and Astronomy Research Council, and formerly by the UK Science and Engineering Research Council
} 


\section{Introduction}

In the standard model (SM) the particle interactions conserve lepton flavour, although there is no underlying symmetry supporting this feature. However, experimental evidence for lepton flavour violation (LFV) in solar and atmospheric neutrino oscillations has been reported $[1,2]$. The experimental upper bounds on neutrino masses imply very small LFV effects in the charged lepton sector. The observation of such effects would clearly indicate new phenomena beyond the SM.

In $e p$ collisions at HERA, LFV processes $e p \rightarrow \mu X$ or $e p \rightarrow \tau X$ lead to final states with a muon or a tau and a hadronic system $X$. The LFV process can proceed via the exchange of a leptoquark (LQ), a boson with both lepton and baryon quantum number which appears naturally as a colour triplet scalar or vector boson in many extensions of the SM such as grand unified theories [3-5], supersymmetry $[6,7]$, compositeness $[8,9]$ and technicolor [10-13].

In this paper a search for LFV phenomena is performed in ep collision data recorded during the years 1998-2000 by the $\mathrm{H} 1$ experiment, corresponding to an integrated luminosity of $66.5 \mathrm{pb}^{-1}$ for $e^{+} p$ collisions and $13.7 \mathrm{pb}^{-1}$ for $e^{-} p$ collisions at a centre-of-mass energy $\sqrt{s}=319 \mathrm{GeV}$. The present results supercede those derived in previous searches at the $\mathrm{H} 1$ experiment using $e^{+} p$ collisions at $\sqrt{s}=$ $300 \mathrm{GeV}[14]$.

\section{Experimental conditions}

A detailed description of the $\mathrm{H} 1$ detector can be found in $[15,16]$. In the following, only the detector components relevant for this analysis are briefly discussed. The origin of the $\mathrm{H} 1$ coordinate system is the nominal $e p$ interaction point, with the direction of the proton beam defining the positive $z$-axis (forward direction). Transverse momenta and azimuthal angles are measured in the $x y$ plane. The pseudorapidity is related to the polar angle $\theta$ by $\eta=-\ln \tan (\theta / 2)$.

A tracking system consisting of central and forward detectors is used to measure charged particle trajectories and to determine the interaction vertex. The central tracker is composed of two concentric cylindrical drift chambers pro-

\footnotetext{
f Supported by FNRS-FWO-Vlaanderen, IISN-IIKW and IWT and by Interuniversity Attraction Poles Programme, Belgian Science Policy

g Partially Supported by the Polish State Committee for Scientific Research, PBS/DESY/70/2006

h Supported by VEGA SR grant no. 2/7062/27

i Supported by the Swedish Natural Science Research Council

j Supported by CONACYT, México, grant 400073-F

k Partially Supported by Russian Foundation for Basic Research, grants 03-02-17291 and 04-02-16445

${ }^{1}$ Supported by the Deutsche Forschungsgemeinschaft

$m$ Supported by the Ministry of Education of the Czech Republic under the projects LC527 and INGO-1P05LA259

${ }^{n}$ Supported by the Swiss National Science Foundation
}

viding full acceptance for particles in the range $22^{\circ}<\theta<$ $160^{\circ}$, complemented by a silicon vertex detector [17] covering the range $30^{\circ}<\theta<150^{\circ}$. Transverse momenta $\left(P_{\mathrm{T}}\right)$ are determined in the central region from the curvature of the particle trajectories in a magnetic field of $1.15 \mathrm{~T}$ with an effective resolution of $\sigma\left(P_{\mathrm{T}}\right) / P_{\mathrm{T}} \simeq 0.01 P_{\mathrm{T}}(\mathrm{GeV})$. The tracking is complemented in the forward region $7^{\circ}<\theta<$ $25^{\circ}$ by a system of drift chambers perpendicular to the beam axis.

With a polar coverage of $4^{\circ}<\theta<154^{\circ}$ and full azimuthal acceptance, the liquid argon (LAr) calorimeter encloses the tracking chambers. It consists of an inner electromagnetic part with a fine granularity and an outer hadronic part with a coarser granularity. The energy resolution of the LAr calorimeter for electrons and hadrons was determined in test beam measurements to be $\sigma / E=12 \% / \sqrt{E(\mathrm{GeV})} \oplus 1 \%$ and $\sigma / E=50 \% / \sqrt{E(\mathrm{GeV})} \oplus$ $2 \%$, respectively $[18,19]$. In the backward region $153^{\circ}<$ $\theta<178^{\circ}$, the LAr calorimeter is complemented by a leadscintillating fibre spaghetti calorimeter.

The iron return yoke of the magnet is instrumented with streamer tubes to identify muon tracks. Further chambers of the central muon system are positioned around the yoke to provide a precise muon track measurement in the polar range $5^{\circ}<\theta<175^{\circ}$. Additional drift chambers positioned at either side of a toroidal magnet are employed to detect muons in the forward direction $\left(3^{\circ}<\theta<17^{\circ}\right)$.

The luminosity is determined from the rate of the Bethe-Heitler process $e p \rightarrow e p \gamma$, measured using a photon detector located close to the beam pipe at $z=-103 \mathrm{~m}$.

Electrons are identified as compact and isolated electromagnetic clusters in the calorimeter. Within the acceptance of the tracking detectors, an associated track is required. A muon candidate is identified by associating an isolated track in the forward muon system or in the inner tracking system with a track segment or an energy deposit in the instrumented iron. The hadronic final state is reconstructed from the deposits in the LAr calorimeter in combination with tracking information. The hadrons are then combined into jets using the inclusive $k_{\mathrm{T}}$-algorithm [20] with a $P_{\mathrm{T}}$-weighted recombination scheme where jets are treated as massless and the separation parameter is set to one.

\section{LFV phenomenology and SM background processes}

The LFV processes $e p \rightarrow \mu X$ and $e p \rightarrow \tau X$ can be attributed to LQs produced at HERA predominantly by electron-quark fusion.

In the framework of the Buchmüller-Rückl-Wyler (BRW) effective model [21], LQs are classified into 14 types with respect to the quantum numbers spin, isospin and chirality. Leptoquarks carry both lepton $(L)$ and baryon $(B)$ quantum numbers. The fermion number $F=L+3 B$ is assumed to be conserved, taking values of $F=2$ for $e^{-} q$ processes and $F=0$ for $e^{+} q$ processes. Leptoquark pro- 

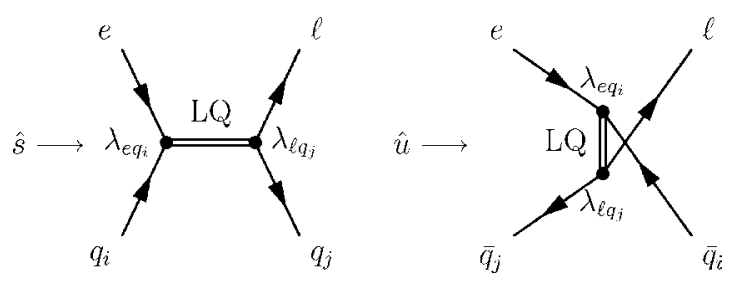

Fig. 1. Left: $s$-channel resonant LQ production and decay to a lepton-quark pair. Right: $u$-channel exchange of a LQ. The indices $i$ and $j$ represent quark generation indices, such that $\lambda_{e q_{i}}$ denotes the coupling of an electron to a quark of generation $i$, and $\lambda_{\ell q_{j}}$ is the coupling of the outgoing lepton $\ell$ to a quark of generation $j$. For $\ell=\mu, \tau$, the LQ introduces LFV

cesses proceed via $s$ channel resonant LQ production or $u$ channel virtual LQ exchange, as shown in Fig. 1. For LQ masses $m_{\mathrm{LQ}}$ well below the $e^{ \pm} p$ centre-of-mass energy, the $s$ channel production of $F=2(F=0)$ LQs in $e^{-} p\left(e^{+} p\right)$ collisions dominates. For LQ masses above $319 \mathrm{GeV}$ the $s$ and $u$ channel processes become of equal importance and both $e^{-} p$ and $e^{+} p$ collisions have similar sensitivity to virtual effects from $F=2$ LQs as well as from $F=0$ LQs.

The BRW model assumes lepton flavour conservation (LFC) such that the LQs produced in $e p$ collisions decay only to $e X$ or $\nu_{e} X$ final states. These LQs are referred to in the following as first generation LQs and have been studied in a recent $\mathrm{H} 1$ publication [22]. A general extension of the BRW model allows for the decay of LQs to final states containing a lepton of a different flavour, i.e. $\mu$ or $\tau$, and a jet, as illustrated in Fig. 1. Non-zero couplings $\lambda_{e q_{i}}$ to an electron-quark pair and $\lambda_{\mu q_{j}}\left(\lambda_{\tau q_{j}}\right)$ to a muon(tau)-quark pair are assumed. The indices ${ }^{1} i$ and $j$ represent quark generation indices, such that $\lambda_{e q_{i}}$ denotes the coupling of an electron to a quark of generation $i$, and $\lambda_{\ell q_{j}}$ is the coupling of the outgoing lepton $\ell$ to a quark of generation $j$.

The double differential cross section for the $s$-channel tree level process is [21]:

$$
\begin{aligned}
\frac{\mathrm{d}^{2} \sigma_{s}}{\mathrm{~d} x \mathrm{~d} y} & =\underbrace{\frac{1}{32 \pi \hat{s}}}_{\text {phase space }} \underbrace{\frac{\lambda_{e q}^{2} \lambda_{\ell q}^{2} \hat{s}^{2}}{\left(\hat{s}^{2}-m_{\mathrm{LQ}}^{2}\right)^{2}+m_{\mathrm{LQ}}^{2} \Gamma_{\mathrm{LQ}}^{2}}}_{\text {Breit-Wigner LQ propagator term }} \underbrace{q_{i}(x, \hat{s})}_{\text {parton density }} \\
& \times \begin{cases}\frac{1}{2} & \text { scalar LQ, } \\
2(1-y)^{2} & \text { vector LQ, }\end{cases}
\end{aligned}
$$

where $x$ is the Bjørken scaling variable, $y$ denotes the inelasticity of the $e p$ scattering process, $\hat{s}=s x$ represents the square of the $e q$ centre-of-mass energy and $\Gamma_{\mathrm{LQ}}$ is the total LQ width. A similar expression holds for the $u$ channel exchange [21].

An overview of the extended effective model for the LQ coupling to $u$ and $d$ quarks is given in Table 1. For convenience only one LFV transition is considered: either between the first and the second generations or between the first and the third generations. The branching ratio

\footnotetext{
1 In the following the quark generation indices are attached only when it is relevant.
}

$$
\begin{aligned}
& \mathrm{LQ} \rightarrow \mu(\tau) q \text { is given by } \\
& \mathrm{BR}=\beta_{\ell} \beta_{\mathrm{LFV}} \quad \text { with } \beta_{\mathrm{LFV}}=\frac{\Gamma_{\mu(\tau) q}}{\Gamma_{\mu(\tau) q}+\Gamma_{e q}} \\
& \text { and } \Gamma_{\ell q}=m_{\mathrm{LQ}} \lambda_{\ell q}^{2} \times \begin{cases}\frac{1}{16 \pi} & \text { scalar LQ, } \\
\frac{1}{24 \pi} & \text { vector LQ },\end{cases}
\end{aligned}
$$

where $\Gamma_{\ell q}$ denotes the partial LQ decay width to a lepton $\ell=e, \mu, \tau$ and a quark $q$ and where $\beta_{\ell}=\Gamma_{\ell q} /\left(\Gamma_{\ell q}+\Gamma_{\nu_{\ell} q}\right)$ is the fraction of decays into charged leptons. Some LQs, namely $S_{0}^{L}, S_{1}^{L}, V_{0}^{L}$ and $V_{1}^{L}$, can decay to a neutrino-quark pair resulting in $\beta_{\ell}=0.5$. Since neutrino flavours cannot be distinguished with the $\mathrm{H} 1$ experiment, such final states are not covered in this search, but they are implicitly included in the search for first generation LQs [22].

To determine the signal detection efficiencies, events with LQs are generated using the LEGO [23] event generator with the CTEQ5L parametrisation of the parton distribution functions (PDF) of the proton [24]. The LQ signal expectation is a function of the LQ type, mass, coupling constant and $\beta_{\mathrm{LFV}}$. The analysis usually requires a large number of simulated signal Monte Carlo (MC) samples. To overcome this technical difficulty, the LEGO program is used to produce a high statistics MC signal event sample generated according to a double-differential cross section $\mathrm{d}^{2} \sigma_{\text {generic }} /\left(\mathrm{d} x \mathrm{~d} Q^{2}\right)$ obtained from (1) by replacing the Breit-Wigner LQ propagator term with a constant. This unique MC sample is used to calculate the efficiency to select a LQ of a given type, mass $m_{\mathrm{LQ}}$, coupling $\lambda_{e q}$ and $\beta_{\mathrm{LFV}}$ by attributing to each event a weight:

$$
\begin{aligned}
& w\left(\text { type, } m_{\mathrm{LQ}}, \lambda_{e q}, \beta_{\mathrm{LFV}}, x, Q^{2}\right) \\
& =\frac{\frac{\mathrm{d}^{2} \sigma_{\text {exact }}}{\mathrm{d} x \mathrm{~d} Q^{2}}\left(\text { type }, m_{\mathrm{LQ}}, \lambda_{e q}, \beta_{\mathrm{LFV}}, x, Q^{2}\right)}{\frac{\mathrm{d}^{2} \sigma_{\text {generic }}}{\mathrm{d} x \mathrm{~d} Q^{2}}}\left(x, Q^{2}\right)
\end{aligned}
$$

where $Q^{2}=s x y$ refers to the generated negative momentum transfer squared and $x$ is the Bjørken scaling variable known at the generator level. This procedure provides an exact prediction over the full range of LQ production parameters and avoids approaches like the narrow width approximation or the high mass (contact interaction) approximation.

The LQ kinematics are reconstructed using the double angle method [25]. The direction of the detected lepton and jet are used to reconstruct the Bjørken scaling variable $x$ and therefore the LQ mass $m_{\mathrm{LQ}}^{\mathrm{rec}}=\sqrt{x s}$.

The contributions from standard model (SM) background processes which may mimic the signal include neutral current (NC) and charged current (CC) deep-inelastic scattering (DIS), photoproduction, lepton pair production and real $W$ boson production. These processes are briefly described below:

\section{- NC DIS $(e p \rightarrow e X)$}

NC DIS processes contribute to the selected event sample if the scattered electron is attributed to the tau 
Table 1. The 14 leptoquark (LQ) types of the Buchmüller-Rückl-Wyler classification [21] in the Aachen notation [23]. The LQ subscripts refer to the weak isospin and the superscripts refer to the lepton chirality. Columns 2-4 display the spin $J$, fermion number $F$ and electrical charge $Q$. The dominant resonant production process in $e p$ scattering and the corresponding coupling is shown in columns 5 and 6 respectively. Leptoquarks which couple to a left-handed lepton doublet and can decay into a neutrino-quark pair, have a charged lepton decay branching ratio of $\beta_{\ell}=\Gamma_{\ell q} /\left(\Gamma_{\ell q}+\Gamma_{\nu_{\ell} q}\right)=1 / 2$

\begin{tabular}{|c|c|c|c|c|c|c|}
\hline Type & $J$ & $F$ & $Q$ & $e p$ dominant process & Coupling & $\begin{array}{c}\text { Branching } \\
\text { ratio } \beta_{\ell}\end{array}$ \\
\hline \multirow{2}{*}{$S_{0}^{L}$} & \multirow{2}{*}{0} & \multirow{2}{*}{2} & \multirow{2}{*}{$-1 / 3$} & \multirow[b]{2}{*}{$e_{L}^{-} u_{L} \longrightarrow\{$} & $\lambda_{L}$ & $1 / 2$ \\
\hline & & & & & $-\lambda_{L}$ & $1 / 2$ \\
\hline$S_{0}^{R}$ & 0 & 2 & $-1 / 3$ & $e_{R}^{-} u_{R} \longrightarrow \ell^{-} u$ & $\lambda_{R}$ & 1 \\
\hline$\widetilde{S}_{0}^{R}$ & 0 & 2 & $-4 / 3$ & $e_{R}^{-} d_{R} \longrightarrow \ell^{-} d$ & $\lambda_{R}$ & 1 \\
\hline \multirow{3}{*}{$S_{1}^{L}$} & \multirow{3}{*}{0} & \multirow{3}{*}{2} & \multirow{2}{*}{$-1 / 3$} & \multirow{2}{*}{$e_{L}^{-} u_{L} \longrightarrow\{$} & $-\lambda_{L}$ & $1 / 2$ \\
\hline & & & & & $-\lambda_{L}$ & $1 / 2$ \\
\hline & & & $-4 / 3$ & $e_{L}^{-} d_{L} \longrightarrow \ell^{-} d$ & $-\sqrt{2} \lambda_{L}$ & 1 \\
\hline$V_{1 / 2}^{L}$ & 1 & 2 & $-4 / 3$ & $e_{L}^{-} d_{R} \longrightarrow \ell^{-} d$ & $\lambda_{L}$ & 1 \\
\hline \multirow[t]{2}{*}{$V_{1 / 2}^{R}$} & \multirow[t]{2}{*}{1} & \multirow[t]{2}{*}{2} & $-1 / 3$ & $e_{R}^{-} u_{L} \longrightarrow \ell^{-} u$ & $\lambda_{R}$ & 1 \\
\hline & & & $-4 / 3$ & $e_{R}^{-} d_{L} \longrightarrow \ell^{-} d$ & $\lambda_{R}$ & 1 \\
\hline$\widetilde{V}_{1 / 2}^{L}$ & 1 & 2 & $-1 / 3$ & $e_{L}^{-} u_{R} \longrightarrow \ell^{-} u$ & $\lambda_{L}$ & 1 \\
\hline \multirow{2}{*}{$V_{0}^{L}$} & \multirow{2}{*}{1} & \multirow{2}{*}{0} & \multirow{2}{*}{$+2 / 3$} & \multirow{2}{*}{$e_{R}^{+} d_{L} \longrightarrow\left\{\begin{array}{l}\ell^{\prime} d \\
\bar{\nu}_{\ell} u\end{array}\right.$} & $\lambda_{L}$ & $1 / 2$ \\
\hline & & & & & $\lambda_{L}$ & $1 / 2$ \\
\hline$V_{0}^{R}$ & 1 & 0 & $+2 / 3$ & $e_{L}^{+} d_{R} \longrightarrow \ell^{+} d$ & $\lambda_{R}$ & 1 \\
\hline$\widetilde{V}_{0}^{R}$ & 1 & 0 & $+5 / 3$ & $e_{L}^{+} u_{R} \longrightarrow \ell^{+} u$ & $\lambda_{R}$ & 1 \\
\hline \multirow{3}{*}{$V_{1}^{L}$} & \multirow{3}{*}{1} & \multirow{3}{*}{0} & \multirow{2}{*}{$+2 / 3$} & \multirow{2}{*}{$e_{R}^{+} d_{L} \longrightarrow\{$} & $-\lambda_{L}$ & $1 / 2$ \\
\hline & & & & & $\lambda_{L}$ & $1 / 2$ \\
\hline & & & $+5 / 3$ & $e_{R}^{+} u_{L} \longrightarrow \ell^{+} u$ & $\sqrt{2} \lambda_{L}$ & 1 \\
\hline$S_{1 / 2}^{L}$ & 0 & 0 & $+5 / 3$ & $e_{R}^{+} u_{R} \longrightarrow \ell^{+} u$ & $\lambda_{L}$ & 1 \\
\hline \multirow[t]{2}{*}{$S_{1 / 2}^{R}$} & \multirow[t]{2}{*}{0} & \multirow[t]{2}{*}{0} & $+2 / 3$ & $e_{L}^{+} d_{L} \longrightarrow \ell^{+} d$ & $-\lambda_{R}$ & 1 \\
\hline & & & $+5 / 3$ & $e_{L}^{+} u_{L} \longrightarrow \ell^{+} u$ & $\lambda_{R}$ & 1 \\
\hline$\widetilde{S}_{1 / 2}^{L}$ & 0 & 0 & $+2 / 3$ & $e_{R}^{+} d_{R} \longrightarrow \quad \ell^{+} d$ & $\lambda_{L}$ & 1 \\
\hline
\end{tabular}

electronic decay or if it is misidentified as a narrow jet corresponding to a tau decay to hadrons. The NC DIS background is modelled using the event generator RAPGAP [26]. The proton PDFs are parametrised using CTEQ5L [24] and hadronisation is performed using JETSET [27] parton showers and the Lund string fragmentation.

- $\mathrm{CC}$ DIS $(e p \rightarrow \nu X)$

Lepton flavour violating processes usually exhibit an imbalance in the measured calorimetric transverse momentum due to either the presence of a minimally ionising muon in $\mu X$ final states or the escaping neutrino(s) from tau decays in $\tau X$ events. This imbalance is exploited in the event selection. The CC DIS process leads to events with genuine missing transverse momentum and therefore contributes to the selected sample if hadrons or photons from the final state are misidentified as muons or if tau decays are falsely reconstructed. The
CC DIS contribution is modelled using the DJANGO event generator [28].

- Photoproduction $(\gamma p \rightarrow X)$

Events from photoproduction processes may contribute to the final selection if a hadron is wrongly identified as a muon or if a narrow hadronic jet fakes the tau signature. This contribution is calculated using the event generator PYTHIA [29]. CTEQ5L [24] serves as the proton PDF parametrisation and the photonic parton distribution parametrisation GRV-LO [30] is used. As PYTHIA only contains leading order $2 \rightarrow 2$ processes, the multi-jet production cross section is underestimated [31]. Therefore, the prediction is scaled up by a factor 1.2 in this analysis, in agreement with previous analyses of jets in photoproduction [31].

- Lepton-pair production $\left(e p \rightarrow e \ell^{+} \ell^{-} X\right)$

Lepton-pair production events contribute to the background because they may lead to high momentum lep- 
tons in the final state. In particular, inelastic di-muon events with one unidentified muon may fake the $\mu X$ LFV signature. The background samples include $e e, \mu \mu$ and $\tau \tau$ production generated with the event generator GRAPE [32].

- $W$ production $(e p \rightarrow e W X)$

Real $W$ boson production leads to final states with isolated high $P_{\mathrm{T}}$ leptons and missing transverse momentum. The simulated $W$ production samples are created with the event generator EPVEC [33] and include leptonic $\left(e \bar{\nu}_{e}, \mu \bar{\nu}_{\mu}, \tau \bar{\nu}_{\tau}\right)$ and hadronic $W$ decays.

All signal and SM samples are passed through a detailed simulation of the $\mathrm{H} 1$ detector response based on the GEANT program [34] and the same reconstruction and analysis algorithms as used for the data.

\section{High $P_{T}$ muon signatures}

Leptoquarks with couplings to the first and the second lepton generation can be produced in $e p$ collisions and may decay to a muon and a quark. The signature is an isolated high $P_{\mathrm{T}}$ muon back-to-back to the hadronic system in the transverse plane. In general, a muon deposits a very small fraction of its energy in the LAr calorimeter. The signal is therefore expected to exhibit large $P_{\mathrm{T}}^{\text {calo }}$, which is the net transverse momentum reconstructed from all clusters recorded in the LAr calorimeter alone.

The event preselection requires at least one muon with a transverse momentum above $10 \mathrm{GeV}$ in the polar angular range $10-140^{\circ}$ and at least one jet. The muon is required to be isolated. The angular distance, $D=\sqrt{(\Delta \eta)^{2}+(\Delta \phi)^{2}}$, of the muon to the nearest track and to the nearest jet is required to be greater than 0.5 and 1.0 , respectively. Only events with $P_{\mathrm{T}}^{\text {calo }}$ greater than $12 \mathrm{GeV}$ are selected. In order to further exploit the event topology in the transverse plane, the cut $V_{\text {ap }} / V_{\mathrm{p}}<0.3$ is employed, where $V_{\mathrm{ap}} / V_{\mathrm{p}}$ is defined as the ratio of the anti-parallel to parallel projections of all energy deposits in the calorimeter with respect to the direction of $P_{\mathrm{T}}^{\text {calo }}$ [35].

Figure 2 displays the distributions of the transverse momentum of the muon, its polar angle $\theta_{\mu}, P_{\mathrm{T}}^{\text {calo }}$ and the acoplanarity $\Delta \phi_{\mu-X}$ between the muon and the hadronic final state $X$ after the muon preselection. The data passing the preselection are well described by the SM prediction. The signal corresponding to a scalar LQ with $m_{\mathrm{LQ}}=$ $200 \mathrm{GeV}$ is also shown. It displays muons with large $P_{\mathrm{T}}^{\mu}$ produced predominantly in the forward direction (low $\theta_{\mu}$ ) in events with significant $P_{\mathrm{T}}^{\text {calo }}$ and back-to-back topology $\Delta \phi_{\mu-X} \simeq 180^{\circ}$.

In the final LFV selection step, the NC DIS background is further suppressed by rejecting events with identified electrons, and by accepting only events with an imbalance of the calorimeter deposits, $P_{\mathrm{T}}^{\text {calo }}>25 \mathrm{GeV}$, and with a back-to-back topology, $\Delta \phi_{\mu-X}>170^{\circ}$. The latter selection criterion is only applied for events for which the
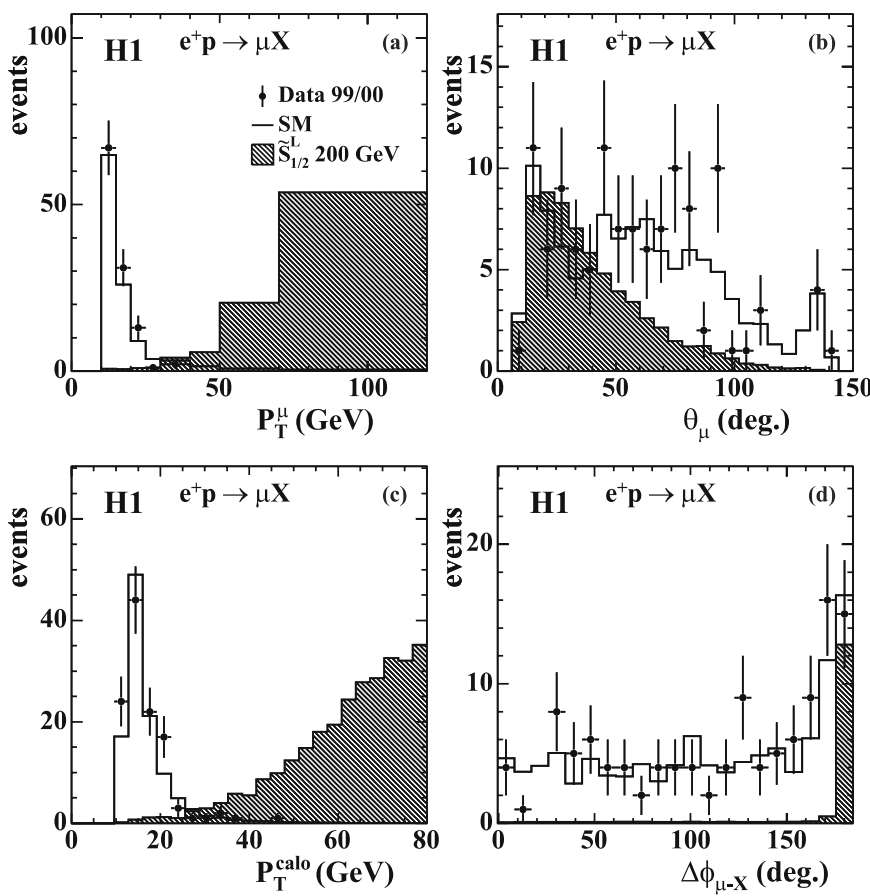

Fig. 2. Control distributions of the preselected $\mu X$ sample: a muon transverse momentum, b muon polar angle, $\mathbf{c}$ transverse momentum as measured from the calorimeter deposits and $\mathbf{d}$ acoplanarity between the muon and the hadronic final state $X$. Data (points) from $e^{+} p$ collisions are compared to the SM expectation (histogram). The LFV signal MC sample of a leptoquark $\widetilde{S}_{1 / 2}^{L}$ with $m_{\mathrm{LQ}}=200 \mathrm{GeV}$ and $\lambda_{e q}=\lambda_{\mu q}=$ 0.3 is shown hatched with arbitrary normalisation in each plot

hadronic final state is well contained in the detector, with the reconstructed polar angle $7^{\circ}<\theta_{X}<140^{\circ}$.

The selection efficiency ranges from $40 \%$ to $60 \%$ depending on the LQ mass and type (see Table 2).

\section{High $P_{\top}$ tau signatures}

Leptoquarks with couplings to the first and the third lepton generation can be produced in $e p$ collisions and may decay to a tau and a quark. Tau leptons are identified using the electronic, muonic and hadronic decays of the tau.

\subsection{Electronic tau decays}

The final state resulting from the electronic tau decay, $\tau \rightarrow e \nu_{e} \nu_{\tau}$, leads to an event topology that is very similar to that of high $Q^{2}$ NC DIS events. The preselection follows that presented in [36]. A reconstructed jet with a minimal transverse momentum of $P_{\mathrm{T}}^{j}>25 \mathrm{GeV}$ back-to-back in the transverse plane to an electron with $P_{\mathrm{T}}^{e}>10 \mathrm{GeV}$ is required. The kinematic domain is restricted to $Q^{2}>$ $1000 \mathrm{GeV}^{2}$ and $y>0.1$. Figure 3 a shows the distribution of 
Table 2. Summary of the selection results of the search for the LFV processes $e p \rightarrow \mu X$ and $e p \rightarrow \tau X$. The results of the individual tau decay channels are also shown. The errors on the SM MC expectation include statistical and systematic errors added in quadrature. Examples of signal selection efficiencies for leptoquarks of the types $S_{0}^{R}, V_{1 / 2}^{L}, V_{0}^{R}$ and $S_{1 / 2}^{L}$ coupling to quarks from the first generation and with masses $m_{\mathrm{LQ}}$ of $150 \mathrm{GeV}$ and $500 \mathrm{GeV}$ are also shown. For the tau decay channels the efficiencies are normalised to the sum of all tau decays

\begin{tabular}{|c|c|c|c|c|c|c|c|c|}
\hline \multicolumn{9}{|c|}{ H1: Search for LFV $\left(e^{-} p: \quad 13.7 \mathrm{pb}^{-1}, \quad e^{+} p: \quad 66.5 \mathrm{pb}^{-1}\right)$} \\
\hline \multirow{2}{*}{\multicolumn{2}{|c|}{ Channel }} & \multicolumn{2}{|c|}{ Selection results } & \multirow[b]{2}{*}{$m_{\mathrm{LQ}}$} & \multicolumn{3}{|c|}{ Selection efficiency } & \multirow[b]{2}{*}{$\widetilde{S}_{1 / 2}^{L}$} \\
\hline & & Data & SM MC & & $S_{0}^{R}$ & $V_{1 / 2}^{L}$ & $V_{0}^{R}$ & \\
\hline \multirow[t]{3}{*}{$e p \rightarrow \mu X$} & $e^{-} p$ & 0 & $0.18 \pm 0.06$ & $150 \mathrm{GeV}$ & $58.0 \%$ & $60.9 \%$ & $60.1 \%$ & $57.7 \%$ \\
\hline & & & & $500 \mathrm{GeV}$ & $47.2 \%$ & $38.5 \%$ & $42.3 \%$ & $37.8 \%$ \\
\hline & $e^{+} p$ & 0 & $1.03 \pm 0.32$ & $150 \mathrm{GeV}$ & $55.5 \%$ & $57.9 \%$ & $58.7 \%$ & $55.8 \%$ \\
\hline \multirow[t]{4}{*}{$e p \rightarrow \tau X$} & $e^{-} p$ & 0 & $0.75 \pm 0.21$ & $150 \mathrm{GeV}$ & $28.3 \%$ & $27.6 \%$ & $27.1 \%$ & $28.1 \%$ \\
\hline & & & & $500 \mathrm{GeV}$ & $21.3 \%$ & $14.4 \%$ & $17.1 \%$ & $13.8 \%$ \\
\hline & $e^{+} p$ & 1 & $4.90 \pm 0.85$ & $150 \mathrm{GeV}$ & $26.8 \%$ & $26.4 \%$ & $26.9 \%$ & $27.0 \%$ \\
\hline & & & & $500 \mathrm{GeV}$ & $17.0 \%$ & $16.7 \%$ & $14.1 \%$ & $17.3 \%$ \\
\hline $\begin{array}{l}e p \rightarrow \tau X \\
\hookrightarrow \tau \rightarrow e \nu_{e} \nu_{\tau}\end{array}$ & $e^{-} p$ & 0 & $0.28 \pm 0.19$ & $150 \mathrm{GeV}$ & $9.0 \%$ & $7.8 \%$ & $7.6 \%$ & $8.9 \%$ \\
\hline \multirow{4}{*}{$\begin{array}{l}e p \rightarrow \tau X \\
\hookrightarrow \tau \rightarrow \mu \nu_{\mu} \nu_{\tau}\end{array}$} & $e^{-} p$ & 0 & $0.18 \pm 0.06$ & $150 \mathrm{GeV}$ & $7.4 \%$ & $7.6 \%$ & $7.6 \%$ & $7.4 \%$ \\
\hline & & & & $500 \mathrm{GeV}$ & $6.3 \%$ & $4.7 \%$ & $5.4 \%$ & $4.6 \%$ \\
\hline & $e^{+} p$ & 0 & $1.03 \pm 0.32$ & $150 \mathrm{GeV}$ & $7.8 \%$ & $8.0 \%$ & $8.1 \%$ & $7.8 \%$ \\
\hline & & & & $500 \mathrm{GeV}$ & $5.2 \%$ & $5.2 \%$ & $4.5 \%$ & $5.3 \%$ \\
\hline \multirow{4}{*}{$\begin{array}{l}e p \rightarrow \tau X \\
\hookrightarrow \tau \rightarrow h \nu_{\tau}\end{array}$} & $e^{-} p$ & 0 & $0.29 \pm 0.06$ & $150 \mathrm{GeV}$ & $11.9 \%$ & $12.2 \%$ & $11.9 \%$ & $11.8 \%$ \\
\hline & & & & $500 \mathrm{GeV}$ & $8.3 \%$ & $5.7 \%$ & $6.5 \%$ & $5.4 \%$ \\
\hline & $e^{+} p$ & 1 & $2.63 \pm 0.57$ & $150 \mathrm{GeV}$ & $10.7 \%$ & $11.2 \%$ & $11.5 \%$ & $10.8 \%$ \\
\hline & & & & $500 \mathrm{GeV}$ & $7.0 \%$ & $6.4 \%$ & $5.6 \%$ & $6.7 \%$ \\
\hline
\end{tabular}

$P_{\mathrm{T}}^{\text {miss }}$ after this preselection, where $P_{\mathrm{T}}^{\text {miss }}$ is defined as the total missing transverse momentum reconstructed from all observed particles.

In the final selection a large missing transverse momentum $P_{\mathrm{T}}^{\text {miss }}>20 \mathrm{GeV}$ is required in order to account for the expected missing momentum carried by the neutrinos produced in the tau decay. These neutrinos are boosted along the electron direction, implying an imbalance between the transverse momenta of the electron $P_{\mathrm{T}}^{e}$ and the hadronic final state $P_{\mathrm{T}}^{X}$. Hence, the restriction $P_{\mathrm{T}}^{e} / P_{\mathrm{T}}^{X}<0.8$ further reduces NC DIS background. In addition, the azimuthal distance between the missing transverse momentum and the electron must not exceed $20^{\circ}$. The remaining NC DIS background, due to mismeasured electron energies leading to missing energy near the electron, is reduced by the requirement $P_{\mathrm{T}}^{e-\text { clu }} / P_{\mathrm{T}}^{e \text {-trk }}>0.7$, where $P_{\mathrm{T}}^{e \text {-clu }}$ is measured from the electromagnetic cluster and $P_{\mathrm{T}}^{e \text {-trk }}$ from tracking information. Any events with additional isolated muons are excluded from the electronic tau decay channel. The final selection in the electronic tau decay channel yields an efficiency normalised to all tau decays of $3 \%-10 \%$, which is limited by the branching fraction $\operatorname{BR}\left(\tau \rightarrow e \nu_{e} \nu_{\tau}\right)=$ $17.8 \%$ [37] and dependent on the assumed LQ mass and type (see Table 2).

\subsection{Muonic tau decays}

Muonic tau decays $\tau \rightarrow \mu \nu_{\mu} \nu_{\tau}$ result in similar final states as the high $P_{\mathrm{T}}$ muon signatures described in Sect. 4. The same selection cuts described therein are applied here. To account for possible effects due to different muon kinematics resulting from a tau decay, the selection efficiency was studied in detail with a LFV MC signal sample with a $\tau X$ final state and a subsequent muonic tau decay. The selection efficiency ranges between $4 \%$ and $8 \%$, which is dependent on the LQ mass and type, normalised to all tau decays and limited by $\operatorname{BR}\left(\tau \rightarrow \mu \nu_{\mu} \nu_{\tau}\right)=17.4 \%$ [37] (see Table 2).

\subsection{Hadronic tau decays}

The hadronic decays of the high $P_{\mathrm{T}}$ tau lead to a typical signature of a high $P_{\mathrm{T}}$ "pencil-like" jet. The signal topology is a di-jet event with no leptons. The tau-jet is characterised by a narrow energy deposit in the calorimeter and low track multiplicity with predominantly one or three tracks in the identification cone of the jet. The neutrino from the tau decay are boosted along the direction of the hadrons. The missing transverse momentum in the event is aligned with the tau-jet. 

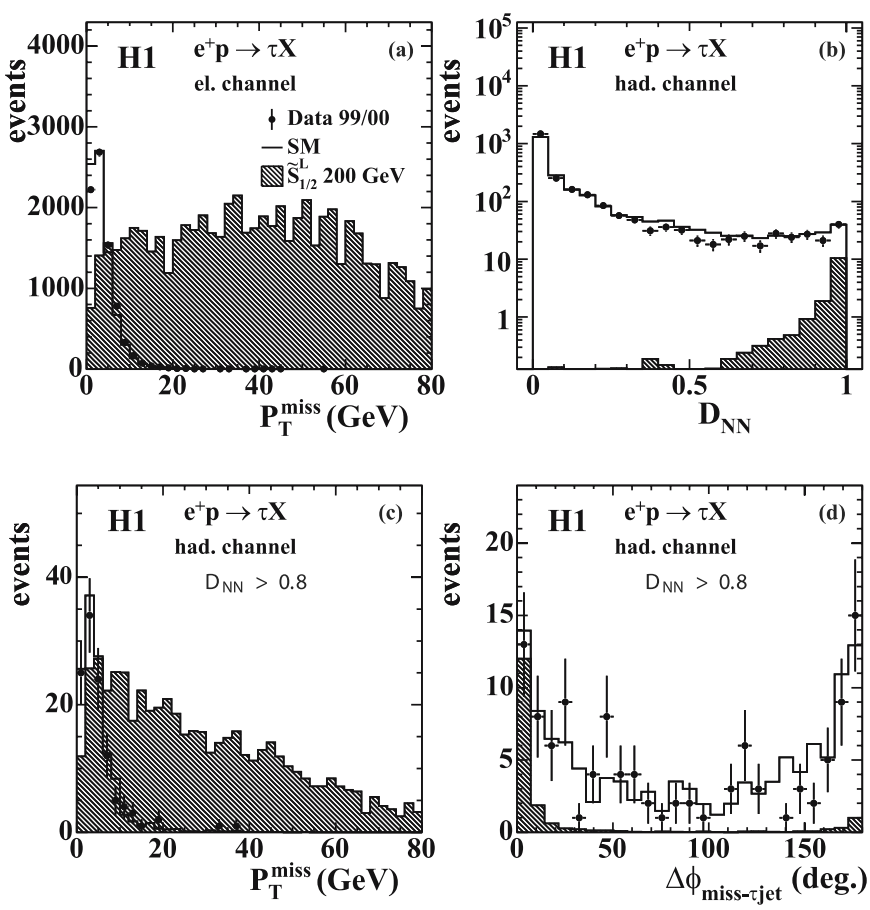

Fig. 3. Distributions of the preselected $\tau X$ sample: a missing transverse momentum in the electronic tau decay channel and $\mathbf{b}$ neural net tau-jet discriminant after the preselection in the hadronic tau decay channel. The restricted sample obtained after the additional cut $\mathcal{D}_{\mathrm{NN}}>0.8$ in the hadronic channel: c missing transverse momentum and $\mathbf{d}$ acoplanarity between the tau-jet and the missing transverse momentum. The LFV signal MC sample of a leptoquark $\widetilde{S}_{1 / 2}^{L}$ with $m_{\mathrm{LQ}}=200 \mathrm{GeV}$ and $\lambda_{e q}=\lambda_{\tau q}=0.3$ is shown hatched with arbitrary normalisation in each plot

Tau-jet candidates are defined as jets with exactly one or three tracks in the tau-jet cone with an opening angle that varies between $5^{\circ}$ and $30^{\circ}$ with decreasing jet momentum. The tracks are required not to be associated with identified electrons or muons and the scalar sum of their transverse momenta is required to be larger than $2 \mathrm{GeV}$. The fine granularity of the LAr calorimeter is used to match extrapolated tracks with energy deposits in the calorimeter and to separate additional neutral particles associated to the tau candidate from unmatched energy deposits in the tau-jet cone. The sum of the four-vectors of the tracks and of the neutral particles defines the tau-jet candidate four-vector.

In the preselection step at least two jets with a transverse momentum $P_{\mathrm{T}}^{\mathrm{jet} 1}>20 \mathrm{GeV}$ and $P_{\mathrm{T}}^{\text {jet } 2}>15 \mathrm{GeV}$ reconstructed in the polar angle range $7^{\circ}<\theta_{\text {jets }}<145^{\circ}$ are required. One jet must fulfil the criteria of a tau-jet candidate with $\theta_{\tau \text { jet }}>20^{\circ}$. In addition, the calorimetric shower shape and tracking signature are exploited to validate the tau-jet candidates. The following estimators are used to separate a tau-jet from quark or gluon induced jets: the number of all tracks associated to the tau-jet candidate, the distance in $\eta-\phi$ between tracks and calorimetric clusters, the number of calorimeter cells of the taujet $n_{\text {cells }}$, the radial extension of the calorimetric deposits $\langle r\rangle=\sum_{i=1}^{n_{\text {cells }}} E_{i} r_{i} / \sum_{i} E_{i}$, the standard deviation $\sigma(r)=$ $\sqrt{\left\langle r^{2}\right\rangle-\langle r\rangle^{2}}$ and the invariant tau-jet mass reconstructed from calorimeter cells. A neural net algorithm is employed and trained using the six estimator variables, as explained in [38]. The neural net yields a discriminator variable $\mathcal{D}_{\mathrm{NN}}$ in the range $0 \leq \mathcal{D}_{\mathrm{NN}} \leq 1$ with values close to 0 for quark or gluon induced jets and close to 1 for hadronic tau decays. The distribution of the discriminant $\mathcal{D}_{\mathrm{NN}}$ after the preselection is depicted in Fig. $3 \mathrm{~b}$. The distributions of $P_{\mathrm{T}}^{\text {miss }}$ and $\Delta \phi_{\text {miss- } \tau \text { jet }}$ after requiring $\mathcal{D}_{\mathrm{NN}}>0.8$ are shown in Fig. $3 \mathrm{c}$ and d. This requirement yields a signal efficiency of $80 \%$ and a quark or gluon induced jet rejection of $95 \%$. After all preselection criteria 16 (112) events are selected in $e^{-} p\left(e^{+} p\right)$ data sample for $22.0 \pm 1.0$ (stat.) (121.1 \pm 5.3(stat.)) expected from the SM.

The final selection step in the hadronic tau decay channel makes use of the characteristic large missing transverse momentum carried by the tau neutrino which is expected to be in the direction of the tau-jet. The difference in $\phi$ between the missing transverse momentum vector and the tau-jet, $\Delta \phi_{\text {miss- } \tau \text { jet }}$, is required to be below $20^{\circ}$. A minimal value of $P_{\mathrm{T}}^{\text {miss }}>12 \mathrm{GeV}$ is chosen for an accurate determination of the direction. In addition $P_{\mathrm{T}}^{\text {calo }}>12 \mathrm{GeV}$ is required. The final signal selection efficiency in the hadronic tau decay channel varies between $3 \%$ and $13 \%$, normalised to all tau decays and limited by the branching fraction $\operatorname{BR}\left(\tau^{-} \rightarrow \nu_{\tau}+\right.$ hadrons $)=64.8 \%$ [37] (see Table 2 ).

\section{Systematic uncertainties}

The following experimental systematic uncertainties are considered:

- The energy of electrons is measured with a systematic uncertainty in the range from $0.7 \%$ to $3 \%$ depending on the polar angle. The uncertainty of the electron direction is estimated to be less than $3 \mathrm{mrad}$ in $\theta$ and $1 \mathrm{mrad}$ in $\phi$.

- The scale uncertainty on the transverse momentum of high $P_{\mathrm{T}}$ muons amounts to $5 \%$. The uncertainty on the reconstruction of the muon direction is $3 \mathrm{mrad}$ in $\theta$ and $1 \mathrm{mrad}$ in $\phi$.

- For the hadronic final state, an energy scale uncertainty of $2 \%$ and a direction uncertainty of $20 \mathrm{mrad}$ are assumed.

- The luminosity of the analysed datasets is known to $1.5 \%$.

The effects of these systematic uncertainties on the signal and the expected SM background are evaluated by shifting the relevant quantities in the MC simulation by their uncertainty and adding all resulting variations in quadrature.

Systematic errors accounting for normalisation uncertainties on the expected background determined from the individual MC event generators are estimated to be $10 \%$ for NC DIS and lepton-pair production, $15 \%$ for $W$ production and $30 \%$ for photoproduction and CC DIS. The relatively large error of $30 \%$ on photoproduction and CC DIS is due to uncertainties on higher-order corrections. 
The errors associated to the background normalisation are added in quadrature to the experimental error to calculate the total error of the SM prediction.

The main theoretical uncertainty on the signal cross section originates from the parton densities. This uncertainty is estimated as described in [22]. It is found to be $5 \%$ for LQs coupling to up-type quarks and varies between $7 \%$ at low masses and $30 \%$ at masses around $290 \mathrm{GeV}$ for LQs coupling to down-type quarks. The correlation between different channels is taken into account for the statistical interpretation and limit calculation [39]. A detailed description of the analysis can be found in [40].

\section{Results}

No candidate is found in the final data sample of the muon channel. The expected number of SM background events is $1.03 \pm 0.32$ in the $e^{+} p$ set and $0.18 \pm 0.06$ in the $e^{-} p$ sample. The largest contribution to this background comes from muon-pair production and the muonic decays of $W$ bosons. These results apply equally to the muonic tau decay channel.

In the electronic tau decay channel no data event is found compared to a SM expectation of $0.28 \pm 0.19$ events in the $e^{-} p$ sample and $1.24 \pm 0.55$ events in the $e^{+} p$ data. NC DIS events with a mismeasured electron energy are the largest background contribution.

No $e^{-} p$ data event passes the final selection criteria in the hadronic tau decay channel. The expected SM background amounts to $0.29 \pm 0.06$. One event is selected in the $e^{+} p$ data for an expected SM prediction of $2.63 \pm 0.57$, dominated by NC DIS and photoproduction processes.

The results of the final selection in all channels are summarised in Table 2 . Typical signal selection efficiencies for some LQ types with a mass of $150 \mathrm{GeV}$ and $500 \mathrm{GeV}$ are also given. The observation is in agreement with the SM prediction and no evidence for LFV is found by the present analysis. Limits on the model parameters presented in Sect. 3 are calculated as described in the following section.

\section{Limits}

The results of the search are interpreted in terms of exclusion limits on the mass and the coupling of LQs that may mediate LFV. The LQ production mechanism at HERA involves non-zero coupling to the first generation fermions $\lambda_{e q}>0$. The LFC decays LQ $\rightarrow e q$ or $\mathrm{LQ} \rightarrow \nu_{e} q$ are therefore possible. In order to cover the full LQ decay width and to generalise the results of LFV searches in $e p$ collisions to an arbitrary weight between the LFC and LFV decay channels, the searches for LFC decays presented in [22] are combined with each of the LFV search channels $\mu X$ or $\tau X$ of the present analysis. It is assumed that only one of the couplings $\lambda_{\mu q}$ and $\lambda_{\tau q}$ is non-zero and therefore the $\mu X$ and $\tau X$ channels enter the limits calculation separately. A modified frequentist method with a likelihood ratio as the test statistic is used to combine the individual data sets and search channels [41].

In first generation LQ signals are searched for in about 400 bins in the $m_{\mathrm{LQ}}-y$ plane and the observed data is in agreement with the irreducible SM NC and $\mathrm{CC}$ background [22]. For the LFV channel $\mu q(\tau q)$, the couplings $\lambda_{e q}$ and $\lambda_{\mu q}\left(\lambda_{\tau q}\right)$ and the LQ mass determine the total production cross section, which is compared to the selected data from the LFV search channel and the first generation results. A combined test statis-
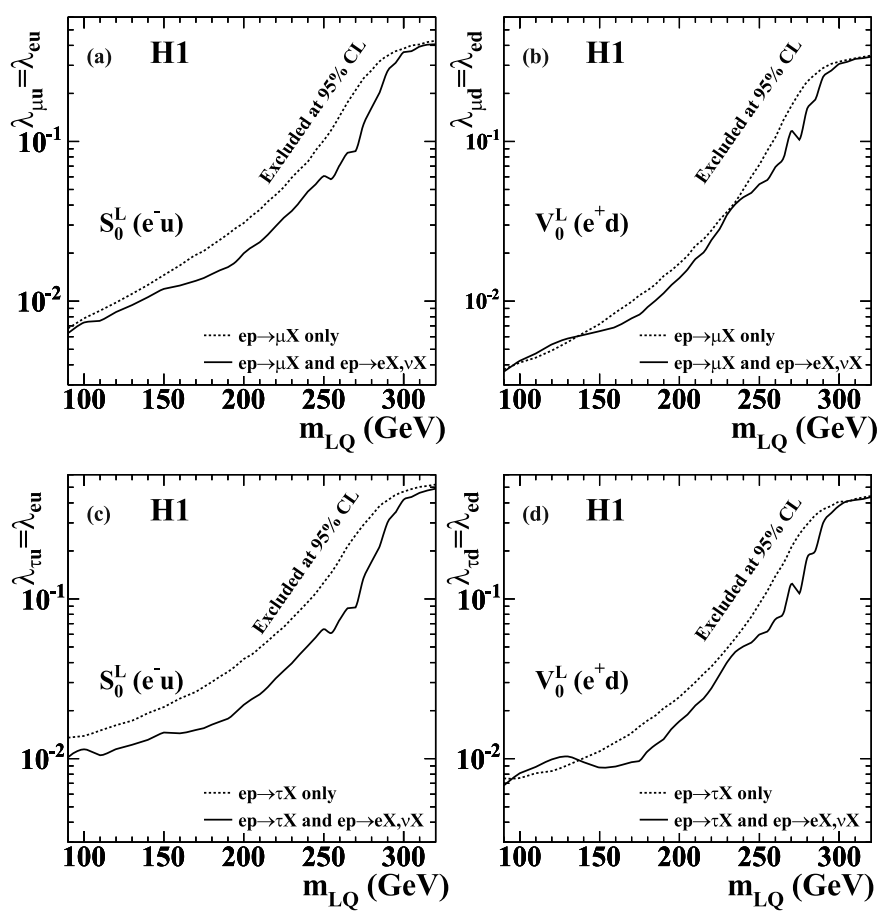

Fig. 4. Comparison of limits at $95 \%$ CL on the coupling constants $\lambda_{\ell q}$ under the assumption $\lambda_{\ell q}=\lambda_{e q}$ as a function of the leptoquark mass $m_{\mathrm{LQ}}$ for: a $S_{0}^{L}$ on $\lambda_{\mu u}=\lambda_{e u}$, b $V_{0}^{L}$ on $\lambda_{\mu d}=\lambda_{e d}$, c $S_{0}^{L}$ on $\lambda_{\tau u}=\lambda_{e u}$, and $\mathbf{d} V_{0}^{L}$ on $\lambda_{\tau d}=\lambda_{e d}$. The areas above the dashed lines represent the exclusion regions using only the lepton flavour violating leptoquark decay channels $e p \rightarrow \mu X$ and $e p \rightarrow \tau X$, respectively. The limits after combination with the results of the search for first generation leptoquarks are shown as solid lines

Table 3. Lower exclusion limits at $95 \% \mathrm{CL}$ on leptoquark masses $m_{\mathrm{LQ}}$ assuming $\lambda_{\mu q}=\lambda_{e q}=0.3$ or $\lambda_{\tau q}=\lambda_{e q}=0.3$

\begin{tabular}{lccccccc}
\hline H1 & \multicolumn{7}{c}{ lower exclusion limits on $m_{\mathrm{LQ}}(\mathrm{GeV})$ at $95 \%$ CL } \\
\hline$F=0$ & $S_{1 / 2}^{L}$ & $S_{1 / 2}^{R}$ & $\widetilde{S}_{1 / 2}^{L}$ & $V_{0}^{L}$ & $V_{0}^{R}$ & $\widetilde{V}_{0}^{R}$ & $V_{1}^{L}$ \\
$e q \rightarrow \mu q$ & 302 & 309 & 288 & 299 & 298 & 333 & 459 \\
$e q \rightarrow \tau q$ & 298 & 298 & 285 & 290 & 293 & 307 & 379 \\
$F=2$ & $S_{0}^{L}$ & $S_{0}^{R}$ & $\widetilde{S}_{0}^{R}$ & $S_{1}^{L}$ & $V_{1 / 2}^{L}$ & $V_{1 / 2}^{R}$ & $\widetilde{V}_{1 / 2}^{L}$ \\
$e q \rightarrow \mu q$ & 294 & 294 & 278 & 306 & 299 & 374 & 336 \\
$e q \rightarrow \tau q$ & 293 & 294 & 276 & 295 & 282 & 302 & 297 \\
\hline
\end{tabular}




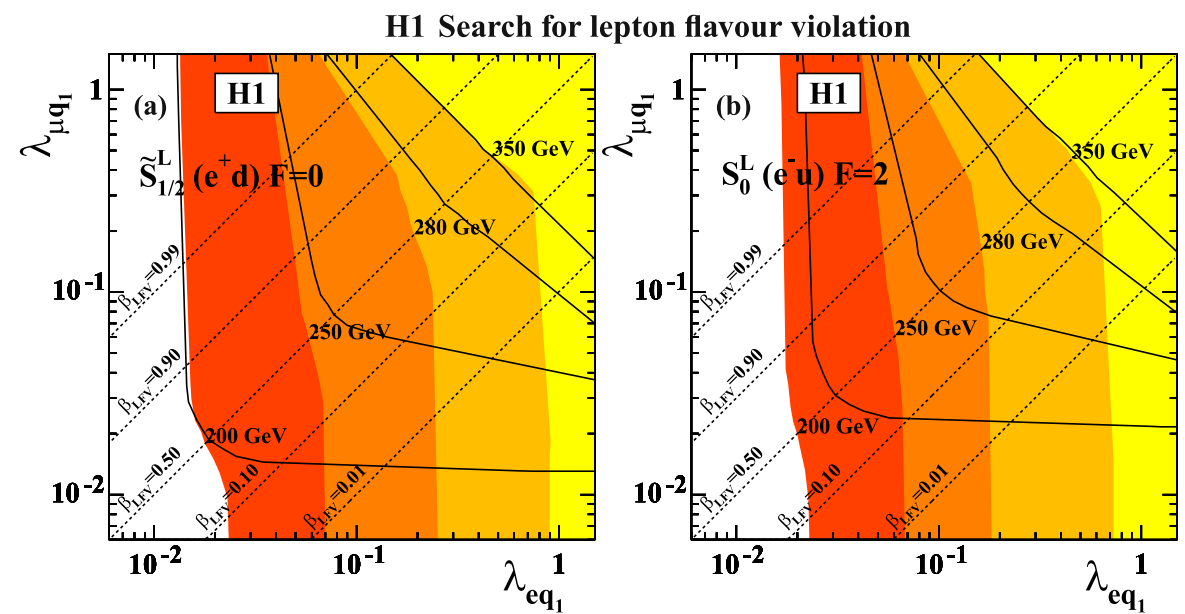

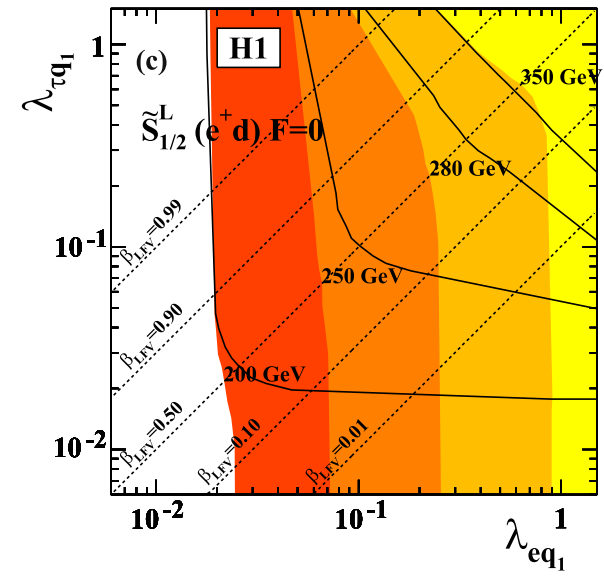

H1 Search for lepton flavour violation

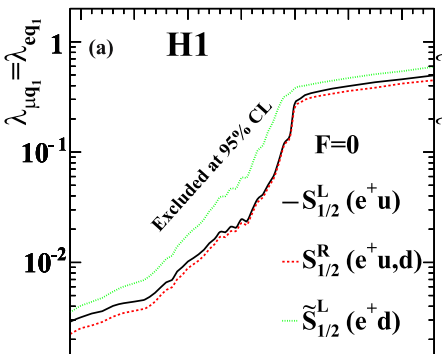

$10_{100}^{-3} 150200250300350400$
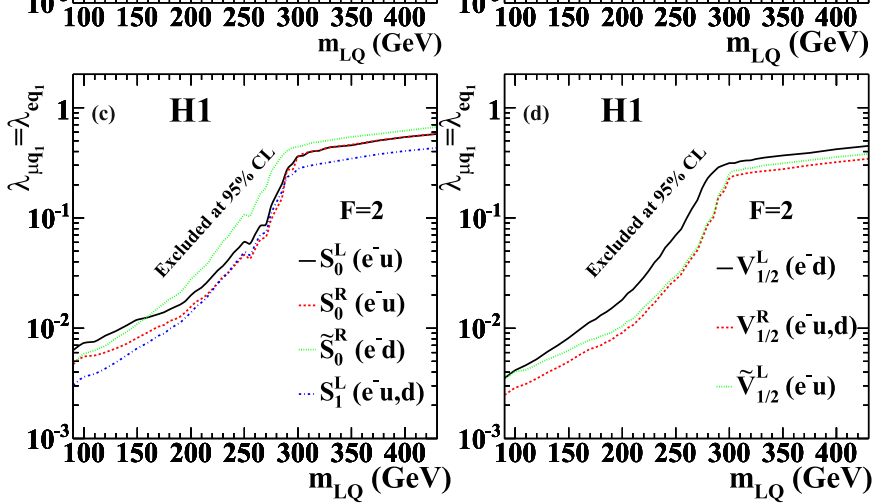

Fig. 6. Limits on the coupling constants $\lambda_{\mu q_{1}}=\lambda_{e q_{1}}$ as a function of the leptoquark mass $m_{\mathrm{LQ}}$ for $\mathbf{a}, \mathbf{b} F=0$ and $\mathbf{c}, \mathbf{d} F=2$ scalar and vector leptoquarks. Regions above the lines are excluded at $95 \% \mathrm{CL}$. The notation $q_{1}$ illustrates that only processes involving first generation quarks are considered

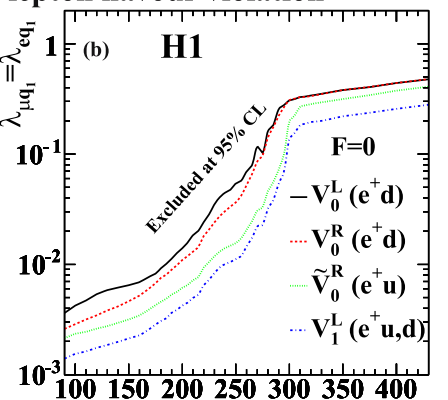

Fig. 5. Excluded regions at $95 \% \mathrm{CL}$ (filled) on $\lambda_{\ell q_{1}}$ as a function of $\lambda_{e q_{1}}$ for four different leptoquark masses. The branching ratio $\beta_{\mathrm{LFV}}=\lambda_{\ell q_{1}}^{2} /\left(\lambda_{\ell q_{1}}^{2}+\lambda_{e q_{1}}^{2}\right)$ is not fixed. Diagonal dashed lines represent iso-curves for fixed values of $\beta_{\mathrm{LFV}}$. The bounds deduced without the combination with first generation leptoquarks are shown as black curves corresponding to the different mass assumptions

\section{H1 Search for lepton flavour violation}
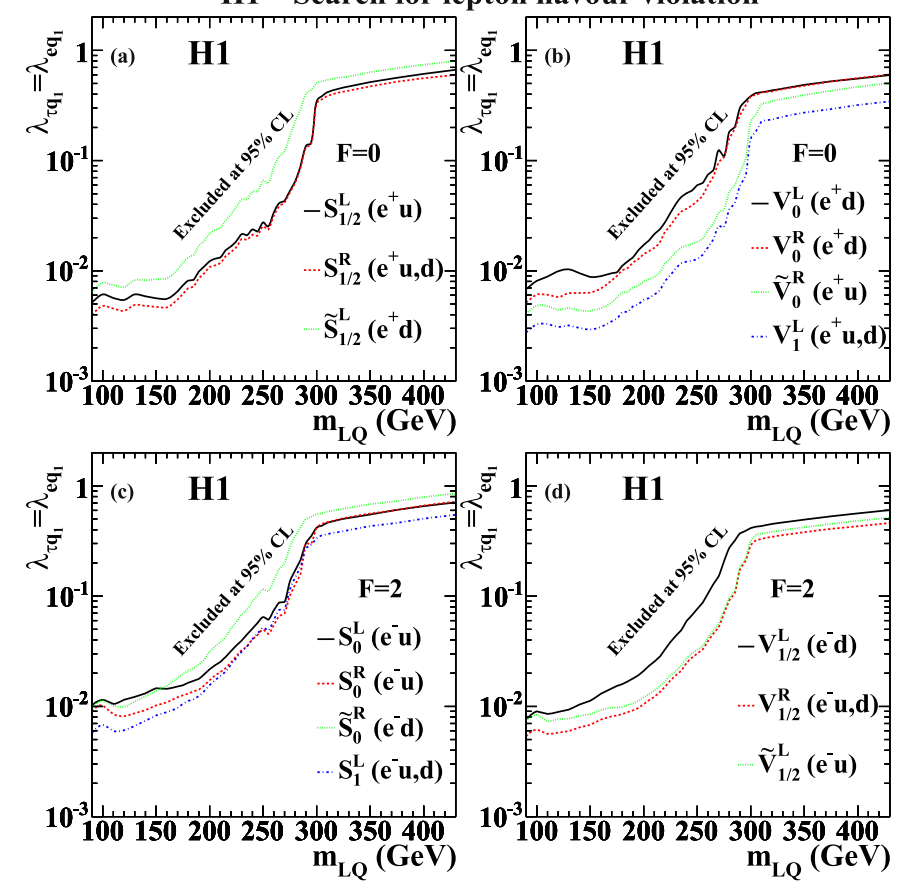

Fig. 7. Limits on the coupling constants $\lambda_{\tau q_{1}}=\lambda_{e q_{1}}$ as a function of the leptoquark mass $m_{\mathrm{LQ}}$ for $\mathbf{a}, \mathbf{b} F=0$ and $\mathbf{c}, \mathbf{d} F=2$ scalar and vector leptoquarks. Regions above the lines are excluded at $95 \% \mathrm{CL}$. The notation $q_{1}$ illustrates that only processes involving first generation quarks are considered 
tic is built and used to set limits as a function of $\lambda_{e q}$, $\lambda_{\mu q}\left(\lambda_{\tau q}\right)$ and $m_{\mathrm{LQ}}$. This procedure implicitly includes in the analysis the decays to a neutrino of any flavour and a quark.

Figure 4 shows limits before and after combination with the search for first generation LQs for the LQ types $S_{0}^{L}$ and $V_{0}^{L}$ up to LQ masses of $320 \mathrm{GeV}$ assuming $\lambda_{e q}=\lambda_{\mu q}$ and $\lambda_{e q}=\lambda_{\tau q}$, i.e. $\beta_{\mathrm{LFV}}=0.5$, in the resonance production region. The comparison for these types exemplifies that the limits on those LQs which can decay to a neutrinoquark pair, namely $S_{0}^{L}, S_{1}^{L}, V_{0}^{L}$ and $V_{1}^{L}$, benefit most from the combination with the search for first generation LQs which covers decays to a neutrino-quark pair. In the high mass regime $m_{\mathrm{LQ}} \gg \sqrt{s}$ (contact interaction region) the obtained limits are similar to those deduced without the combination. The fluctuations in the combined limits are due to the observed data events in the search for first gen- eration LQs. In the mass range from $250 \mathrm{GeV}$ to $300 \mathrm{GeV}$ both the combined limits on $\lambda_{\mu q}$ and $\lambda_{\tau q}$ are for all LQ types up to a factor 2 more stringent than without combination. Table 3 shows the $95 \%$ CL combined lower limits on the LQ mass for all LQ types assuming a coupling of electromagnetic strength $\lambda_{e q}=\lambda_{\mu q}\left(\lambda_{\tau q}\right)=0.3$.

Allowing for an arbitrary decay rate between the LFC and LFV decay channels, $\beta_{\mathrm{LFV}}$, the excluded regions for two LQ types and four mass values in the $\lambda_{\mu q_{1}}-\lambda_{e q_{1}}(\mathrm{a}, \mathrm{b})$ and $\lambda_{\tau q_{1}}-\lambda_{e q_{1}}(\mathrm{c}, \mathrm{d})$ planes are presented in Fig. 5. For very low values of $\beta_{\mathrm{LFV}}\left(\lambda_{e q} \gg \lambda_{\mu q}\left(\lambda_{\tau q}\right)\right)$, the limits on $\lambda_{e q}$ reproduce the bounds published in [22], as expected, since the LFC channel dominates the LQ width. For $\beta_{\mathrm{LFV}} \gg 0.5$ $\left(\lambda_{\mu q}\left(\lambda_{\tau q}\right) \gg \lambda_{e q}\right)$ the present analysis extends significantly the published limits on $\lambda_{e q}$ to lower values. The limit without combination in the contact interaction region (where the cross section is proportional to $\left.\lambda_{e q_{i}} \lambda_{\mu(\tau) q_{j}} / m_{\mathrm{LQ}}^{2}\right)$ forms

Table 4. Limits at 95\% CL on $\lambda_{e q_{i}} \lambda_{\mu q_{j}} / m_{\mathrm{LQ}}^{2}$ for $F=0$ leptoquarks (bold). Combinations of $i$ and $j$ shown in the first column denote the quark generation coupling to the electron and muon respectively. In each cell the first two rows show the process providing the most stringent limit from low energy experiments. The cases marked with ' $*$ ' refer to scenarios involving a top quark, not considered in the present analysis

$$
\text { H1: } e p \rightarrow \mu X, F=0
$$

Upper exclusion limits on $\lambda_{e q_{i}} \lambda_{\mu q_{j}} / m_{\mathrm{LQ}}^{2}\left(\mathrm{TeV}^{-2}\right)$ for lepton flavour violating leptoquarks at $95 \% \mathrm{CL}$

\begin{tabular}{|c|c|c|c|c|c|c|c|}
\hline$q_{i} q_{j}$ & $\begin{array}{l}S_{1 / 2}^{L} \\
e^{-} \bar{u} \\
e^{+} u\end{array}$ & $\begin{array}{c}S_{1 / 2}^{R} \\
e^{-}(\bar{u}+\bar{d}) \\
e^{+}(u+d)\end{array}$ & $\begin{array}{l}\widetilde{S}_{1 / 2}^{L} \\
e^{-} \bar{d} \\
e^{+} d\end{array}$ & $\begin{array}{l}V_{0}^{L} \\
e^{-\bar{d}} \\
e^{+} d\end{array}$ & $\begin{array}{l}V_{0}^{R} \\
e^{-\bar{d}} \\
e^{+} d\end{array}$ & $\begin{array}{l}\widetilde{V}_{0}^{R} \\
e^{-} \bar{u} \\
e^{+} u\end{array}$ & $\begin{array}{c}V_{1}^{L} \\
e^{-}(\sqrt{2} \bar{u}+\bar{d}) \\
e^{+}(\sqrt{2} u+d)\end{array}$ \\
\hline 12 & $\begin{array}{c}D \rightarrow \mu \bar{e} \\
0.8 \\
\mathbf{1 . 4}\end{array}$ & $\begin{array}{c}K \rightarrow \mu \bar{e} \\
2 \times 10^{-5} \\
\mathbf{1 . 2}\end{array}$ & $\begin{array}{c}K \rightarrow \mu \bar{e} \\
2 \times 10^{-5} \\
\mathbf{2 . 0}\end{array}$ & $\begin{array}{c}K \rightarrow \mu \bar{e} \\
1 \times 10^{-5} \\
\mathbf{1 . 5}\end{array}$ & $\begin{array}{c}K \rightarrow \mu \bar{e} \\
1 \times 10^{-5} \\
\mathbf{1 . 5}\end{array}$ & $\begin{array}{c}D \rightarrow \mu \bar{e} \\
0.4 \\
\mathbf{1 . 1}\end{array}$ & $\begin{array}{c}K \rightarrow \mu \bar{e} \\
1 \times 10^{-5} \\
\mathbf{0 . 5}\end{array}$ \\
\hline 13 & $*$ & $\begin{array}{c}B \rightarrow \mu \bar{e} \\
0.1 \\
\mathbf{2 . 1}\end{array}$ & $\begin{array}{c}B \rightarrow \mu \bar{e} \\
0.1 \\
\mathbf{2 . 1}\end{array}$ & $\begin{array}{c}B \rightarrow \mu \bar{e} \\
0.06 \\
\mathbf{1 . 6}\end{array}$ & $\begin{array}{c}B \rightarrow \mu \bar{e} \\
0.06 \\
\mathbf{1 . 6}\end{array}$ & $*$ & $\begin{array}{c}B \rightarrow \mu \bar{e} \\
0.06 \\
\mathbf{1 . 6}\end{array}$ \\
\hline 21 & $\begin{array}{c}D \rightarrow \mu \bar{e} \\
0.8 \\
4.2\end{array}$ & $\begin{array}{c}K \rightarrow \mu \bar{e} \\
2 \times 10^{-5} \\
\mathbf{2 . 9}\end{array}$ & $\begin{array}{c}K \rightarrow \mu \bar{e} \\
2 \times 10^{-5} \\
\mathbf{4 . 1}\end{array}$ & $\begin{array}{c}K \rightarrow \mu \bar{e} \\
1 \times 10^{-5} \\
\mathbf{1 . 7}\end{array}$ & $\begin{array}{c}K \rightarrow \mu \bar{e} \\
1 \times 10^{-5} \\
\mathbf{1 . 7}\end{array}$ & $\begin{array}{c}D \rightarrow \mu \bar{e} \\
0.4 \\
\mathbf{1 . 5}\end{array}$ & $\begin{array}{c}K \rightarrow \mu \bar{e} \\
1 \times 10^{-5} \\
\mathbf{0 . 7}\end{array}$ \\
\hline 23 & $*$ & $\begin{array}{c}B \rightarrow \bar{\mu} e K \\
7.5 \times 10^{-3} \\
\mathbf{5 . 2}\end{array}$ & $\begin{array}{c}B \rightarrow \bar{\mu} e K \\
7.5 \times 10^{-3} \\
\mathbf{5 . 2}\end{array}$ & $\begin{array}{c}B \rightarrow \bar{\mu} e K \\
3.8 \times 10^{-3} \\
\mathbf{3 . 5}\end{array}$ & $\begin{array}{c}B \rightarrow \bar{\mu} e K \\
3.8 \times 10^{-3} \\
\mathbf{3 . 5}\end{array}$ & $*$ & $\begin{array}{c}B \rightarrow \bar{\mu} e K \\
3.8 \times 10^{-3} \\
\mathbf{3 . 5}\end{array}$ \\
\hline 31 & $*$ & $\begin{array}{c}B \rightarrow \mu \bar{e} \\
0.1 \\
\mathbf{5 . 3}\end{array}$ & $\begin{array}{c}B \rightarrow \mu \bar{e} \\
0.1 \\
\mathbf{5 . 3}\end{array}$ & $\begin{array}{c}V_{u b} \\
0.14 \\
\mathbf{1 . 8}\end{array}$ & $\begin{array}{c}B \rightarrow \mu \bar{e} \\
0.06 \\
\mathbf{1 . 8}\end{array}$ & $*$ & $\begin{array}{c}V_{u b} \\
0.14 \\
\mathbf{1 . 8}\end{array}$ \\
\hline 32 & $*$ & $\begin{array}{c}B \rightarrow \bar{\mu} e K \\
7.5 \times 10^{-3} \\
\mathbf{7 . 0}\end{array}$ & $\begin{array}{c}B \rightarrow \bar{\mu} e K \\
7.5 \times 10^{-3} \\
\mathbf{7 . 0}\end{array}$ & $\begin{array}{c}B \rightarrow \bar{\mu} e K \\
3.8 \times 10^{-3} \\
\mathbf{2 . 8}\end{array}$ & $\begin{array}{c}B \rightarrow \bar{\mu} e K \\
3.8 \times 10^{-3} \\
\mathbf{2 . 8}\end{array}$ & $*$ & $\begin{array}{c}B \rightarrow \bar{\mu} e K \\
3.8 \times 10^{-3} \\
\mathbf{2 . 8}\end{array}$ \\
\hline
\end{tabular}


a cross-diagonal straight line following different values of $\beta_{\mathrm{LFV}}$. The combination in the contact interaction region, e.g. $m_{\mathrm{LQ}}=350 \mathrm{GeV}$, barely strengthens the limit as the virtual effects of the high mass LQ contact interaction at low values of $\sqrt{\hat{s}}$ are marginal compared to the irreducible $\mathrm{NC}$ and CC DIS background. Fluctuations of the data may even result in a less stringent combined limit.

Figures 6 and 7 display the 95\% CL upper limits on the coupling $\lambda_{\mu q}$ and $\lambda_{\tau q}$ of all 14 LQ types to a muonquark pair and a tau-quark pair, respectively, as a function of the LQ mass leading to LFV in $e p$ collisions, assuming $\lambda_{e q}=\lambda_{\mu q}\left(\lambda_{\tau q}\right)$. The limit curves referring to the LQ types $S_{0}^{L}$ and $\widetilde{S}_{1 / 2}^{L}$ are identical to profiles of the corresponding excluded regions following the value $\beta_{\mathrm{LFV}}=0.5$ in Fig. 5. The limits are most stringent at low LQ masses with values $\mathcal{O}\left(10^{-3}\right)$ around $m_{\mathrm{LQ}}=100 \mathrm{GeV}$. Corresponding to the steeply falling parton density function for high values of $x$, the LQ production cross section decreases rapidly and exclusion limits are less stringent towards higher LQ masses. For LQ mass values near the kinematical limit of $319 \mathrm{GeV}$, the limit corresponding to a resonantly produced LQ turns smoothly into a limit on the virtual effects of both an off-shell $s$-channel LQ process and a $u$-channel LQ exchange. At masses $m_{\mathrm{LQ}}>\sqrt{s}$ the two processes contract to an effective four-fermion interaction, where the cross section is proportional to $\left(\lambda_{\mu(\tau) q} \lambda_{e q} / m_{\mathrm{LQ}}^{2}\right)^{2}$. This feature is visible in the constant increase of the exclusion limit for masses above the $e p$ centre-of-mass energy of $\sqrt{s}=319 \mathrm{GeV}$. Due to initial state QED radiation and very low parton densities for masses near $\sqrt{s}$ the "kink" of the transition region is shifted to somewhat smaller masses of around $290-300 \mathrm{GeV}$.

It is noticeable that the limits on vector LQs are more stringent compared to those on the scalars, due to the con-

Table 5. Limits at 95\% CL on $\lambda_{e q_{i}} \lambda_{\tau q_{j}} / m_{\mathrm{LQ}}^{2}$ for $F=0$ leptoquarks (bold). Combinations of $i$ and $j$ shown in the first column denote the quark generation coupling to the electron and tau respectively. In each cell the first two rows show the process providing the most stringent limit from low energy experiments. The cases marked with ' $*$ ' refer to scenarios involving a top quark, not considered in the present analysis

$$
\text { H1: } e p \rightarrow \tau X, F=0
$$

Upper exclusion limits on $\lambda_{e q_{i}} \lambda_{\tau q_{j}} / m_{\mathrm{LQ}}^{2}\left(\mathrm{TeV}^{-2}\right)$ for lepton flavour violating leptoquarks at $95 \% \mathrm{CL}$

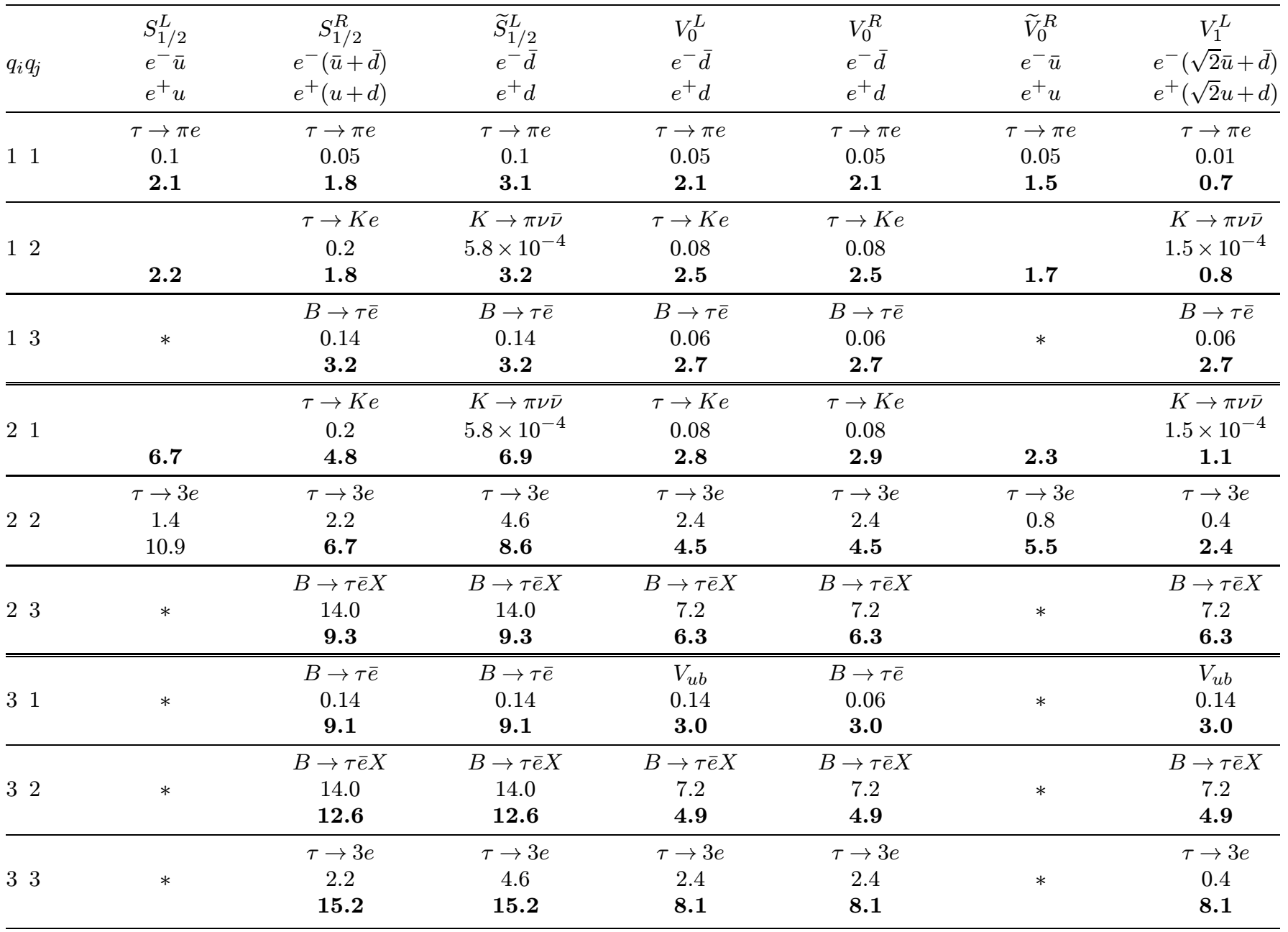


siderably higher cross section and the slightly higher acceptance. In each plot those LQ types that have couplings to both $u$ and $d$ quarks exhibit the best limit. The limits corresponding to LQs coupling to a $u$ quark are more stringent than those corresponding to LQs coupling to the $d$ quark only, as expected from the larger $u$ quark density in the proton. The LQs $S_{0}^{L}$ and $S_{0}^{R}\left(V_{0}^{L}\right.$ and $\left.V_{0}^{R}\right)$ differ only by the decay into a neutrino and a quark of the lefthanded LQ. As this decay channel is not covered in the LFV decay channels, the left-handed LQ cannot be as strictly excluded as the right-handed one. This argument applies to the resonant production where the analysis is only sensitive to the partial width of the LQ. In the high mass region the limits for $S_{0}^{L}$ and $S_{0}^{R}\left(V_{0}^{L}\right.$ and $\left.V_{0}^{R}\right)$ are similar, as the four-fermion interaction is independent of the decay width.

The limits on $\lambda_{\mu(\tau) q}=\lambda_{e q}$ derived from the virtual effects of a $500 \mathrm{GeV} \mathrm{LQ}$ are transformed into a limit on the value $\lambda_{\mu(\tau) q_{j}} \lambda_{e q_{i}} / m_{\mathrm{LQ}}^{2}$ and shown in Tables 4 and 5 for $F=0$ LQs and in Tables 6 and 7 for $F=2$ LQs. For each LQ type the limit is calculated for the hypothesis of a process with only the quarks of flavours $i$ and $j$ involved. With respect to quark flavours, the selection criteria described in Sects. 4 and 5 are inclusive since no flavour tagging of the hadronic jet is used.

These results may be compared with constraints from low energy experiments, based on the non-observation of LFV in muon scattering and rare decays of mesons and leptons [37]. The interpretation in terms of leptoquark exchange and limits on $\lambda_{\mu(\tau) q_{j}} \lambda_{e q_{i}} / m_{\mathrm{LQ}}^{2}[42-44]$ are also shown in Tables $4-7$. Bounds of similar magnitude are observed for processes involving $e \rightarrow \tau$ transitions and charm or bottom quarks. In these cases some of the limits obtained in the present analysis are superior to those from low energy experiments.

Table 6. Limits at 95\% CL on $\lambda_{e q_{i}} \lambda_{\mu q_{j}} / m_{\mathrm{LQ}}^{2}$ for $F=2$ leptoquarks (bold). Combinations of $i$ and $j$ shown in the first column denote the quark generation coupling to the electron and muon respectively. In each cell the first two rows show the process providing the most stringent limit from low energy experiments. The cases marked with '*' refer to scenarios involving a top quark, not considered in the present analysis

$$
\text { H1: } e p \rightarrow \mu X, F=2
$$

Upper exclusion limits on $\lambda_{e q_{i}} \lambda_{\mu q_{j}} / m_{\mathrm{LQ}}^{2}\left(\mathrm{TeV}^{-2}\right)$ for lepton flavour violating leptoquarks at $95 \% \mathrm{CL}$

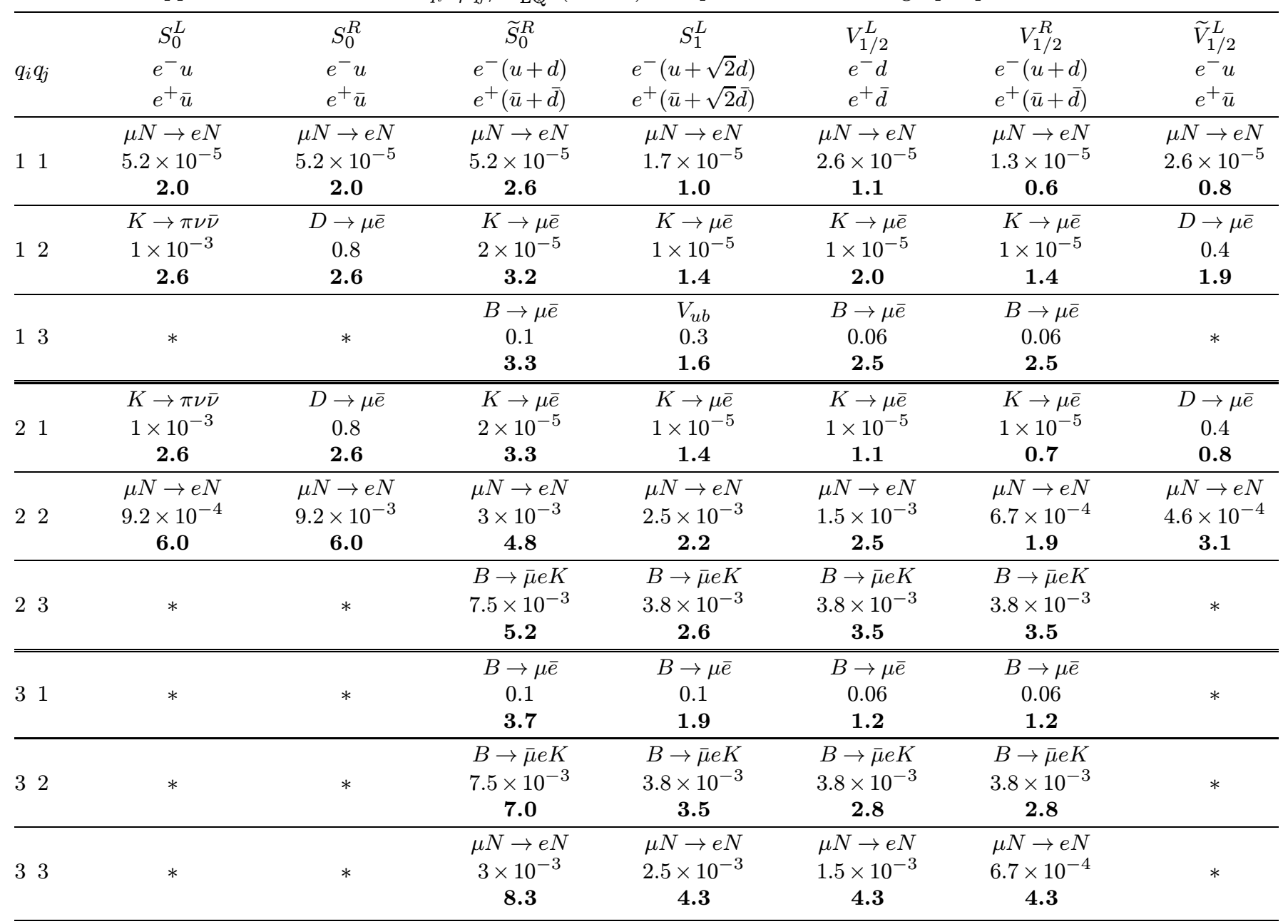


Table 7. Limits at $95 \%$ CL on $\lambda_{e q_{i}} \lambda_{\tau q_{j}} / m_{\mathrm{LQ}}^{2}$ for $F=2$ leptoquarks (bold). Combinations of $i$ and $j$ shown in the first column denote the quark generation coupling to the electron and tau respectively. In each cell the first two rows show the process providing the most stringent limit from low energy experiments. The cases marked with '*' refer to scenarios involving a top quark, not considered in the present analysis

\begin{tabular}{|c|c|c|c|c|c|c|c|}
\hline & Upper e & limits & $\tau q_{j} / m_{\mathrm{LQ}}^{2}$ & $\begin{array}{l}\rightarrow \tau X, F=2 \\
\left.{ }^{2}\right) \text { for lepton } \mathrm{fl}\end{array}$ & or violating l & uarks at 95 & \\
\hline$q_{i} q_{j}$ & $\begin{array}{l}S_{0}^{L} \\
e^{-} u \\
e^{+} \bar{u}\end{array}$ & $\begin{array}{c}S_{0}^{R} \\
e^{-} u \\
e^{+} \bar{u}\end{array}$ & $\begin{array}{c}\widetilde{S}_{0}^{R} \\
e^{-(u+d)} \\
e^{+}(\bar{u}+\bar{d})\end{array}$ & $\begin{array}{c}S_{1}^{L} \\
e^{-(u+\sqrt{2} d)} \\
e^{+}(\bar{u}+\sqrt{2} \bar{d})\end{array}$ & $\begin{array}{l}V_{1 / 2}^{L} \\
e^{-} d \\
e^{+} \bar{d}\end{array}$ & $\begin{array}{c}V_{1 / 2}^{R} \\
e^{-(u+d)} \\
e^{+}(\bar{u}+\bar{d})\end{array}$ & $\begin{array}{l}\widetilde{V}_{1 / 2}^{L} \\
e^{-} u \\
e^{+} \bar{u}\end{array}$ \\
\hline 11 & $\begin{array}{l}G_{\mathrm{F}} \\
0.3 \\
\mathbf{3 . 0}\end{array}$ & $\begin{array}{c}\tau \rightarrow \pi e \\
0.1 \\
\mathbf{3 . 0}\end{array}$ & $\begin{array}{c}\tau \rightarrow \pi e \\
0.1 \\
4.2\end{array}$ & $\begin{array}{c}\tau \rightarrow \pi e \\
0.02 \\
\mathbf{1 . 7}\end{array}$ & $\begin{array}{c}\tau \rightarrow \pi e \\
0.05 \\
\mathbf{1 . 8}\end{array}$ & $\begin{array}{c}\tau \rightarrow \pi e \\
0.02 \\
\mathbf{1 . 0}\end{array}$ & $\begin{array}{c}\tau \rightarrow \pi e \\
0.05 \\
\mathbf{1 . 2}\end{array}$ \\
\hline 12 & $\begin{array}{c}K \rightarrow \pi \nu \bar{\nu} \\
5.8 \times 10^{-4} \\
\mathbf{4 . 0}\end{array}$ & 4.0 & $\begin{array}{c}\tau \rightarrow K e \\
0.2 \\
\mathbf{5 . 0}\end{array}$ & $\begin{array}{c}K \rightarrow \pi \nu \bar{\nu} \\
2.9 \times 10^{-4} \\
\mathbf{2 . 1}\end{array}$ & $\begin{array}{c}K \rightarrow \pi \nu \bar{\nu} \\
2.9 \times 10^{-4} \\
\mathbf{3 . 5}\end{array}$ & $\begin{array}{c}\tau \rightarrow K e \\
0.08 \\
\mathbf{2 . 3}\end{array}$ & 3.1 \\
\hline 13 & $*$ & $*$ & $\begin{array}{c}B \rightarrow \tau \bar{e} \\
0.14 \\
\mathbf{5 . 3}\end{array}$ & $\begin{array}{l}V_{u b} \\
0.3 \\
\mathbf{2 . 7} \\
\end{array}$ & $\begin{array}{c}B \rightarrow \tau \bar{e} \\
0.06 \\
\mathbf{4 . 2}\end{array}$ & $\begin{array}{c}B \rightarrow \tau \bar{e} \\
0.06 \\
\mathbf{4 . 2}\end{array}$ & $*$ \\
\hline 22 & $\begin{array}{c}\tau \rightarrow 3 e \\
1.4 \\
\mathbf{1 0 . 8}\end{array}$ & $\begin{array}{c}\tau \rightarrow 3 e \\
1.4 \\
\mathbf{1 0 . 9}\end{array}$ & $\begin{array}{c}\tau \rightarrow 3 e \\
4.6 \\
\mathbf{8 . 6}\end{array}$ & $\begin{array}{c}\tau \rightarrow 3 e \\
3.8 \\
\mathbf{3 . 9}\end{array}$ & $\begin{array}{c}\tau \rightarrow 3 e \\
2.4 \\
\mathbf{4 . 5} \\
\end{array}$ & $\begin{array}{c}\tau \rightarrow 3 e \\
1.1 \\
\mathbf{3 . 5}\end{array}$ & $\begin{array}{c}\tau \rightarrow 3 e \\
0.8 \\
\mathbf{5 . 5}\end{array}$ \\
\hline 23 & $*$ & $*$ & $\begin{array}{c}\rightarrow \bar{\tau} e X \\
14.0 \\
\mathbf{9 . 3} \\
\end{array}$ & $\begin{array}{c}\rightarrow \bar{\tau} e X \\
7.2 \\
\mathbf{4 . 7}\end{array}$ & $\begin{array}{c}B \rightarrow \bar{\tau} e X \\
7.2 \\
\mathbf{6 . 3}\end{array}$ & $\begin{array}{c}\rightarrow \bar{\tau} e X \\
7.2 \\
\mathbf{6 . 3}\end{array}$ & $*$ \\
\hline $\begin{array}{ll}3 & 1\end{array}$ & $*$ & $*$ & $\begin{array}{c}B \rightarrow \tau \bar{e} \\
0.14 \\
\mathbf{6 . 3}\end{array}$ & $\begin{array}{c}B \rightarrow \tau \bar{e} \\
0.06 \\
\mathbf{3 . 1}\end{array}$ & $\begin{array}{c}B \rightarrow \tau \bar{e} \\
0.06 \\
\mathbf{1 . 9}\end{array}$ & $\begin{array}{c}B \rightarrow \tau \bar{e} \\
0.06 \\
\mathbf{1 . 9}\end{array}$ & * \\
\hline 32 & $*$ & $*$ & $\begin{array}{c}B \rightarrow \bar{\tau} e X \\
14.0 \\
\mathbf{1 2 . 6} \\
\end{array}$ & $\begin{array}{c}B \rightarrow \bar{\tau} e X \\
7.2 \\
\mathbf{6 . 4}\end{array}$ & $\begin{array}{c}B \rightarrow \bar{\tau} e X \\
7.2 \\
\mathbf{4 . 9}\end{array}$ & $\begin{array}{c}\rightarrow \bar{\tau} e X \\
7.2 \\
\mathbf{4 . 9}\end{array}$ & $*$ \\
\hline
\end{tabular}

The results on LFV in LQ production are directly comparable with those from the ZEUS experiment [45]. Similar limits are obtained. At hadron colliders LQs are mainly produced in pairs independently of the coupling, and therefore searches cannot constrain LFV couplings. Lower mass limits on the second and third generation leptoquarks extend up to $250 \mathrm{GeV}$ and $150 \mathrm{GeV}$, respectively, depending on the type and the assumed decay branching ratios [46-49]. Similarly, second and third generation leptoquarks are pair produced in $e^{+} e^{-}$annihilation where typical lower mass bounds reach values of $100 \mathrm{GeV}$ [50].

\section{Conclusion}

A search for lepton flavour violation processes induced by leptoquarks in $e p$ collisions at a centre-of-mass energy of
$319 \mathrm{GeV}$ with the $\mathrm{H} 1$ experiment at HERA is presented. No signal for the LFV processes $e p \rightarrow \mu X$ or $e p \rightarrow \tau X$ is found. Constraints on LFV LQ couplings are set combining the LFV search with the search for first generation LQs. The limits are a factor of 2 to 4 more stringent and extend beyond the domain in LQ mass excluded by previous searches performed by the $\mathrm{H} 1$ experiment [14]. Exclusion limits on several scenarios of LFV transitions of the kind $e q_{i} \rightarrow \tau q_{j}$ are comparable or more stringent than limits from searches for certain rare meson or tau decays. Assuming a coupling of electromagnetic strength, leptoquarks mediating lepton flavour violating processes $e \rightarrow \mu$ and $e \rightarrow \tau$ can be ruled out up to masses of $459 \mathrm{GeV}$ and $379 \mathrm{GeV}$, respectively.

Acknowledgements. We are grateful to the HERA machine group whose outstanding efforts have made this experiment possible. We thank the engineers and technicians for their work in constructing and maintaining the $\mathrm{H} 1$ detector, our funding agencies for financial support, the DESY technical staff for con- 
tinual assistance and the DESY directorate for support and for the hospitality which they extend to the non DESY members of the collaboration.

\section{References}

1. Super-Kamiokande Collaboration, Y. Fukuda et al., Phys. Rev. Lett. 81, 1562 (1998) [hep-ex/9807003]

2. SNO Collaboration, Q.R. Ahmad et al., Phys. Rev. Lett. 89, 011301 (2002) [nucl-ex/0204008]

3. J.C. Pati, A. Salam, Phys. Rev. D 81, 275 (1974)

4. H. Georgi, S.L. Glashow, Phys. Rev. Lett. 32, 438 (1974)

5. P. Langacker, Phys. Rep. 72, 185 (1981)

6. H.P. Nilles, Phys. Rep. 110, 1 (1984)

7. H.E. Haber, G.L. Kane, Phys. Rep. 117, 75 (1985)

8. B. Schrempp, F. Schrempp, Phys. Lett. B 153, 101 (1985)

9. J. Wudka, Phys. Lett. B 167, 337 (1986)

10. S. Dimopoulos, L. Susskind, Nucl. Phys. B 155, 237 (1979)

11. S. Dimopoulos, Nucl. Phys. B 168, 69 (1980)

12. E. Farhi, L. Susskind, Phys. Rev. D 20, 3404 (1979)

13. E. Farhi, L. Susskind, Phys. Rep. 74, 277 (1981)

14. H1 Collaboration, C. Adloff et al., Eur. Phys. J. C 11, 447 (1999) [hep-ex/9907002] [Erratum ibid. 14, 553 (2000)]

15. H1 Collaboration, I. Abt et al., Nucl. Instrum. Methods A 386, 310 (1997)

16. H1 Collaboration, I. Abt et al., Nucl. Instrum. Methods A 386, 348 (1997)

17. D. Pitzl et al., Nucl. Instrum. Methods A 454, 334 (2000) [hep-ex/0002044]

18. H1 Calorimeter Group, B. Andrieu et al., Nucl. Instrum. Methods A 336, 499 (1993)

19. H1 Calorimeter Group, B. Andrieu et al., Nucl. Instrum. Methods A 350, 57 (1994)

20. J.M. Butterworth, J.P. Couchman, B.E. Cox, B.M. Waugh, Comput. Phys. Commun. 153, 85 (2003) [hep-ph/0210022]

21. W. Buchmüller, R. Rückl, D. Wyler, Phys. Lett. B 191, 442 (1987)

22. H1 Collaboration, A. Aktas et al., Phys. Lett. B 629, 9 (2005) [hep-ex/0506044]

23. K. Rosenbauer, dissertation RWTH Aachen (in German), PITHA 95/16, July 1995

24. CTEQ Collaboration, J. Pumplin et al., JHEP 0207, 012 (2002) [hep-ph/0201195]

25. S. Bentvelsen, J. Engelen, P. Kooijman, Proc. Workshop "Physics at HERA" 1991, ed. by W. Buchmüller, G. Ingelman, Vol. 1 (DESY, 1992) p. 23
26. H. Jung, Comput. Phys. Commun. 86, 147 (1995)

27. T. Sjöstrand, Comput. Phys. Commun. 39, 347 (1986)

28. G.A. Schuler, H. Spiesberger, Proc. Workshop "Physics at HERA" 1991, ed. by W. Buchmüller, G. Ingelman, Vol. 3 (DESY, 1992) p. 1419

29. T. Sjöstrand, Comput. Phys. Commun. 82, 74 (1994)

30. M. Glück et al., Phys. Rev. D 46, 1973 (1992)

31. H1 Collaboration, A. Aktas et al., Phys. Lett. B 639, 21 (2006) [hep-ex/0603014]

32. T. Abe, Comput. Phys. Commun. 136, 126 (2001) [hep$\mathrm{ph} / 0012029]$.

33. U. Baur, J. A .M. Vermaseren, D. Zeppenfeld, Nucl. Phys. B 375, 3 (1992)

34. R. Brun et al., CERN-DD/EE-84-1 (1987)

35. H1 Collaboration, C. Adloff et al., Eur. Phys. J. C 19, 269 (2001) [hep-ex/0012052]

36. H1 Collaboration, C. Adloff et al., Eur. Phys. J. C 11, 447 (1999) [hep-ex/9907002] [Erratum ibid. 14, 553 (1999)]

37. Particle Data Group, W.M. Yao et al., J. Phys. G 33, 1 (2006)

38. H1 Collaboration, A. Aktas et al., Eur. Phys. J. C 48, 699 (2006) [hep-ex/0604022]

39. M. Botje, Eur. Phys. J. C 14, 285 (2000) [hep-ph/9912439]

40. L. Lindfeld, Ph.D. Thesis (University of Zürich, 2006), available at

http://www-h1.desy.de/publications/theses_list.html

41. LEP Working Group for Higgs boson searches, R. Barate et al., Phys. Lett. B 565, 61 (2003) [hep-ex/0306033]

42. S. Davidson, D.C. Bailey, B.A. Campbell, Z. Phys. C 61, 613 (1994) [hep-ph/9309310]

43. E. Gabrielli, Phys. Rev. D 62, 055009 (2000) [hep-ph/ 9911539]

44. M. Herz, Diploma thesis (University of Bonn, 2002), hepph/0301079

45. ZEUS Collaboration, S. Chekanov et al., Eur. Phys. J. C 44, 463 (2005) [hep-ex/0501070]

46. D0 Collaboration, V.M. Abazov et al., Phys. Lett. B 636, 183 (2006) [hep-ex/0601047]

47. D0 Collaboration, B. Abbott et al., Phys. Rev. Lett. 81, 38 (1998) [hep-ex/9803009]

48. CDF Collaboration, A. Abulencia et al., Phys. Rev. D 73, 051102 (2006) [hep-ex/0512055]

49. CDF Collaboration, A.A. Affolder et al., Phys. Rev. Lett. 85, 2056 (2000) [hep-ex/0004003]

50. OPAL Collaboration, G. Abbiendi et al., Eur. Phys. J. C 31, 281 (2003) [hep-ex/0305053] 